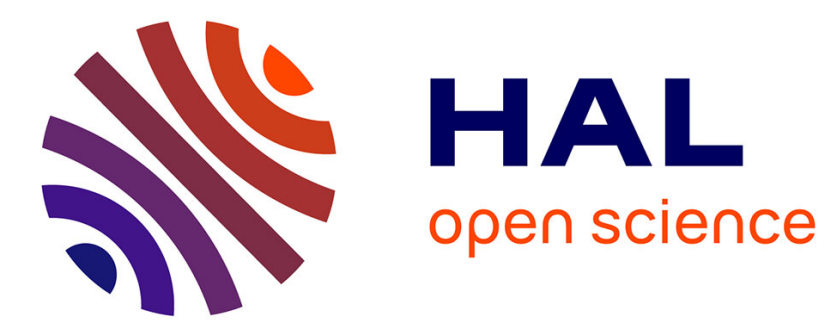

\title{
Synchronization of weakly coupled canard oscillators
}

Elif Köksal Ersöz, Mathieu Desroches, Martin Krupa

\section{To cite this version:}

Elif Köksal Ersöz, Mathieu Desroches, Martin Krupa. Synchronization of weakly coupled canard oscillators. Physica D: Nonlinear Phenomena, 2017, 349, pp.46-61. 10.1016/j.physd.2017.02.016 . hal-01558897

\section{HAL Id: hal-01558897 \\ https://hal.inria.fr/hal-01558897}

Submitted on 11 Jul 2017

HAL is a multi-disciplinary open access archive for the deposit and dissemination of scientific research documents, whether they are published or not. The documents may come from teaching and research institutions in France or abroad, or from public or private research centers.
L'archive ouverte pluridisciplinaire HAL, est destinée au dépôt et à la diffusion de documents scientifiques de niveau recherche, publiés ou non, émanant des établissements d'enseignement et de recherche français ou étrangers, des laboratoires publics ou privés. 


\title{
Synchronization of weakly coupled canard oscillators
}

\author{
Elif Köksal Ersöz ${ }^{\mathrm{a}, *}$, Mathieu Desroches ${ }^{\mathrm{b}}$, Martin Krupa $^{\mathrm{c}}$ \\ ${ }^{a}$ MYCENAE Project Team, Inria Paris, France \\ ${ }^{b}$ MathNeuro Team, Inria Sophia Antipolis Méditerranée, France \\ ${ }^{c}$ Department of Applied Mathematics, University College Cork, Ireland
}

\begin{abstract}
Synchronization has been studied extensively in the context of weakly coupled oscillators using the so-called phase response curve (PRC) which measures how a change of the phase of an oscillator is affected by a small perturbation. This approach was based upon the work of Malkin, and it has been extended to relaxation oscillators. Namely, synchronization conditions were established under the weak coupling assumption, leading to a criterion for the existence of synchronous solutions of weakly coupled relaxation oscillators. Previous analysis relies on the fact that the slow nullcline does not intersect the fast nullcline near one of its fold points, where canard solutions can arise. In the present study we use numerical continuation techniques to solve the adjoint equations and we show that synchronization properties of canard cycles are different than those of classical relaxation cycles. In particular, we highlight a new special role of the maximal canard in separating two distinct synchronization regimes: the Hopf regime and the relaxation regime. Phase plane analysis of slow-fast oscillators undergoing a canard explosion provides an explanation for this change of synchronization properties across the maximal canard.
\end{abstract}

Keywords: canards, phase response curves, slow-fast systems, synchronization, weak coupling

2010 MSC: 34C15, 34C26, 34D06, 34E17, 92C20

\footnotetext{
${ }^{*}$ Corresponding author

Email address: elif.koksal@inria.fr (Elif Köksal Ersöz)
}

Preprint submitted to Elsevier

January 12, 2017 


\section{Introduction}

Synchronization is a research topic of its own, which has produced a large body of knowledge, in particular for so-called weakly coupled oscillators 1, 2, 3 , 4, 5, 6. A classical object of interest in this context is the (infinitesimal) phase

5 phase of an oscillator when applied all along the associated stable limit cycle solution. The derivation of the PRC relies on the linearization of the system along the unperturbed (i.e. uncoupled) cycle and corresponds to the solution of the adjoint variational equation. Solutions to the adjoint problem and PRCs give when the coupling strength is small enough. Such studies are gathered under the name "weakly coupled oscillator theory" 4. This theory has been linked with earlier studies from Malkin [7, 8] by Izhikevich and Hoppensteadt in [4; an explicit proof was given in [9] based on the work of Roseau [10, 11]. to investigate the effects of slowly-varying parameters, underlying bifurcations and coupling strengths on collective dynamics. In one of the pioneering papers on this topic [2], out-of-phase (OP) synchronization (intermediate modes between in-phase (IP) and anti-phase (AP) solutions) was shown to emerge from parameter. A similar bifurcation structure has been found in type-I spiking neuron models, see e.g. [12, 13, 14]. Another recent study related to type-I membranes [15] focused on the transition from IP to OP synchronous states in chains of Wang-Buszaki models coupled by gap junctions. This transition was investigated both analytically and numerically as a function of intrinsic system properties by using phase models and interaction function. In the framework of type-II neuron models, the impact of the Hopf bifurcation on the possible synchronization patterns has been studied, e.g., in [16, 17, 18, 19, 20. Furthermore, variations of the PRC across a Hopf bifurcation were analyzed in 
cortical excitatory neuron models in [21]. Qualitative changes in the behaviour of the PRC were also looked at directly from experimental data in [22] and [23] where the interaction functions were analysed during the transition from Hopf and relaxation oscillators. The existence of different synchronization modes and of bistable regions in weakly coupled slow-fast systems interacting via gap 35 junctions has been underlined in $[24,25,26,27,28,13,29,30,31,32$. Synchronization has also been studied in the context of coupled piecewise linear models, in particular in [33, 34].

Slow-fast oscillators are an important source of complicated dynamics, and particularly in relation to the canard phenomenon [35, 36]. The term canard cycle refers to a class of periodic solutions of slow-fast systems which follow for a long time interval a repelling slow manifold. Canards occur in slow-fast systems near regions of the critical manifold (fast nullsurface) where the key assumption of normal hyperbolicity fails. The most common points of this kind in systems with one slow and one fast variables are generically fold points, so-called canard

45 points. A canard solution flows from an attracting slow manifold to a repelling slow-manifold by passing close to such a canard point. In planar systems, canard cycles exist in a very narrow range of bifurcation parameters, an interval that is exponentially small in the time scale separation parameter $\varepsilon(0<\varepsilon \ll 1)$. These sharp transitions upon parameter variation through the canard regime are called canard explosions [37. Combinations of advanced theoretical techniques, such as blow-up methods [38, and numerical methods [39] have introduced a new understanding of canard-induced complex oscillations in systems with multiple time scales (in $\mathbb{R}^{n}, n \geq 3$ ), in particular mixed-mode oscillations (MMOs) [40] and bursting oscillations [41, 42, and extended their applications to neuroscience [43, 44, 45]. In (weakly) coupled slow-fast systems, the effect of canard solutions has been considered in several aspects such as the formation of clusters, synchrony, phase and amplitude dynamics [46, 47, 48, 49, 50. Recently, canard-mediated variability has been investigated in coupled phantom bursting systems addressing issues on synchronization and desynchronization [51.

In this work we extend previous results on adjoint solutions and weakly cou- 
pled slow-fast oscillators to the case of canard cycles. Analytical formulations of adjoints and interaction functions were studied in [52], which also provides a review of the behavior near bifurcation points. In the framework of relaxation cycles, an expression for the adjoints could be obtained in [53] by taking the 65 singular limit approximation, considering the attracting branches of the critical manifold in place of the slow segments of relaxation cycles, and instantaneous jumps in place of the fast flow. The consequence of using this setup is that the canard regime has not been dealt with. In the present study, we propose an alternative numerical strategy, based on numerical continuation, for the computation of solutions to the adjoint variational equation associated with planar slow-fast systems along a canard explosion.

In parameter space, canards organize the transition between the Hopf regime and the relaxation regime. Therefore we may expect to link the synchronous behavior between these two families [22, 23] by computing adjoints for canard solutions. When performing such computations, we observe a qualitative change in the sign and shape of the adjoint (or equivalently, of the iPRC) near the maximal canard (the cycle with the longest repelling segment). This phenomenon occurs in both canard-explosive systems that we consider here, namely the van der Pol (VDP) oscillator and a two-dimensional (2D) reduction of the Hodgkin80 Huxley $(\mathrm{HH})$ model. We propose an explanation to this qualitative change through the period function of the canard family which has a non-monotonic behavior across the explosion from the Hopf bifurcation point to the relaxation regime, namely, it increases during the headless-canard regime and it decreases during the canard-with-head regime. This remarkable property of canard explosions singles out the maximal (period) canard, for which we highlight a key role in synchronization, which to the best of our knowledge was not reported in previous studies. Similar dependence of the frequency upon a bifurcation parameter has been studied in [54] in the context of "escape-release" mechanisms of central patterns generators. The authors of [55] have then linked this dependence to transitions in PRCs and phase-locking properties occurring in the low-frequency region. 
In the second half of this work, we explore the dependence of the phase difference between the two weakly coupled identical VDP systems on system parameters. By investigating the effect of the main parameter displaying the canard explosion, we observe that the transition in synchronization properties occurring at the maximal canard of the coupled system manifests itself as the AP synchronization state changing its stability through a pitchfork bifurcation in the phase difference. Furthermore, we reveal the presence of $2 n T$-periodic synchronous states in the maximal canard neighborhood appearing via multiple period-doubling (PD) bifurcations. Finally, we consider the effect of the coupling strength on the synchronous states of the oscillators in the maximal canard regime. We give numerical evidence of the presence of $\mathrm{PD}$ cascades not predicted by the theory of weakly coupled oscillators (which is valid for moderate coupling strengths in various systems [14, 56]) but that can be justified using phase plane analysis of the single canard oscillators under scrutiny. We also propose in an analytical formula to compute adjoints associated with limit cycles of slow-fast systems in Liénard systems, which gives satisfactory yet improvable results.

This paper is organized as follows. In Section 2, we introduce the main objects required to compute adjoint solutions along a limit cycle and we present our numerical strategy to do so along families of canard cycles. In Section 3 we analyze numerically the effect of the main parameter controlling the canard explosion on the synchronous states of the coupled VDP system and report a qualitative change occurring near the maximal canard solution. We then explain this change by invoking the properties of the period function associated with such a canard-explosive branch of limit cycles. In Section 4 we focus on the effect of the coupling strength parameter on the synchronous structure in the coupled VDP system near a maximal canard. After concluding and proposing a few perspectives to this work, we present an analytical formula to compute adjoint solutions for the type of systems we have investigated and test this 120 formula numerically in Appendix A 


\section{Computations of adjoint solutions along a family of cycles}

\subsection{Phase Response Curve and Adjoints}

PRCs describe the phase shifts along a stable limit cycle of a dynamical system in response to a stimulus. Weakly coupled oscillator theory [1, 57, 4] is used to predict the phase-locking properties of coupled oscillating system with a "small enough" coupling strength. This theory, which reduces the dynamics of oscillators to a phase variable, implies that coupling has small effects that can accumulate over time and lead to phase-locking behaviors. IPRCs correspond to PRCs in the limit of infinitesimal stimulus. One way to compute iPRCs is by means of non-trivial solutions to the adjoint variational equation associated to the stable limit cycle under consideration; there are numerous other approaches, see e.g. [3, 5].

We now recall the main elements necessary to introduce adjoint solutions associated with a limit cycles. Consider a dynamical system in $\mathbb{R}^{n}$

$$
\frac{d X}{d t}=F(X)
$$

that possesses a $T$-periodic asymptotically stable limit cycle $\gamma$. A phase variable $\phi \in[0, T)$ is defined along the limit cycle $\gamma$ parameterized by time and it is typically normalized to 1 or to $2 \pi$. It can be associated with points on the cycle by writing $\phi=\Theta(x)$ for $x \in \gamma$. Then, perturbing a point $x$ on the limit cycle with corresponding phase $\phi=\Theta(x)$ (which we can also write as $x=X(\phi)$ ) by a small quantity $y \in \mathbb{R}^{n}$ leads to a delay or an advance of the phase. The new phase $\phi^{\prime}$ is given by

$$
\phi^{\prime}=\phi+\nabla_{X} \Theta(x) \cdot y+O\left(\|y\|^{2}\right)
$$

and the difference between the old and new phases for small perturbations are expressed as

$$
\phi^{\prime}-\phi=\nabla_{X} \Theta(x) \cdot y
$$

${ }_{135}$ The vector function $Z$ defined by $\left.Z(\phi)=\nabla_{X} \Theta(X(\phi))\right)$ is the gradient of the phase map describing how infinitesimal perturbations on any system variable 
along the limit cycle changes its phase. The function $Z$ (which depends on $\phi$ or equivalently on $t \in[0, T])$ is the solution of the adjoint variational equation

$$
\frac{d Z(t)}{d t}+A(t)^{T} Z(t)=0
$$

which satisfies the normalization condition

$$
Z(t) \frac{d X_{0}(t)}{d t}=1,
$$

where

$$
A(t)=\left.D_{X} F(X)\right|_{\gamma}
$$
is the linearization of system (1) around the limit cycle $\gamma$. The adjoint equation should be integrated backwards in time to eliminate all the transient components except the periodic one, which gives the solution. An algorithm to compute solutions to adjoint equations, based on backward integration, is embedded in software package XPPAUT [58, or can be coded in MATLAB [59], in addition to a continuation-based approach in MATCont 60].

Canard explosions occur in slow-fast systems in a very narrow parameter range which is exponentially small in the timescale separation parameter $0<\varepsilon \ll 1$. Naturally, this parameter range gets narrower as $\varepsilon$ tends to 0 , and limits the usage of classical tools to compute family of canard orbits and their adjoints. This limit has been acknowledged by Govaerts and Sautois who have introduced a direct numerical approach in the continuation package MATCONT [60. In addition to existing methods, we propose an alternative and simpler continuation-based strategy using the software package AUTO [61]. We formulate a periodic continuation problem which allows us to compute rapidly and reliably a family of cycles with associated non-trivial periodic solution of the adjoint equation. Note that a boundary-value problem (BVP) approach has been proposed in 62], outside of a continuation setup given that the system was with reset. Here, for simplicity, we avoid dealing specifically with boundary conditions and opt for the most natural periodic setting of this numerical problem. An extension of the analytic approach for solving adjoint variational equations in slow-fast systems is given in Appendix A. 


\subsection{Numerical continuation alternative for adjoints}

The numerical continuation approach proposed in the present work allows us to compute limit cycles and associated adjoint solutions along a canard-explosive branch. One of the main advantages of the continuation is the possibility to find solutions in the limit $\varepsilon \rightarrow 0$. We extend the continuation setting of the original system (1), solved in order to find limit cycles, by including equation (2) (once written in first-order form) to find periodic solutions of the adjoint problem along these cycles. In order to compute a limit cycle $\gamma$ together with a periodic solution of the associated adjoint problem along $\gamma$, one needs to solve the following system of equations

$$
\begin{aligned}
\dot{X} & =F(X), \\
\dot{Z} & =-D_{X} F(X)_{\mid \gamma}^{T} Z .
\end{aligned}
$$

Our numerical continuation strategy requires two steps: first, to find a nontrivial solution of the adjoint problem along the (initial) cycle $\gamma$, and second, to follow the extended system (4) (as a periodic continuation problem) in a bifurcation parameter in order to find a branch of such solutions. In the following section we describe these steps by considering two examples of coupled slowfast systems in the canard regime, namely, the VDP system and and a twodimensional reduction of the $\mathrm{HH}$ model for action potential generation whose slow-fast structure and associated canard dynamics were analyzed in [63.

\subsubsection{Adjoint solutions of the VDP system}

In the case of the VDP system, the extended continuation setting (4) reads

$$
\begin{aligned}
x^{\prime} & =y-f(x) \\
y^{\prime} & =\varepsilon(c-x) \\
z_{1}^{\prime} & =f^{\prime}\left(\gamma_{1}(t)\right) z_{1}+\varepsilon z_{2} \\
z_{2}^{\prime} & =-z_{1},
\end{aligned}
$$

with $f(x)=x^{3} / 3-x, 0<\varepsilon \ll 1$ and $c$ is the bifurcation parameter displaying the canard explosion. The system given in (5) merges the original VDP system 

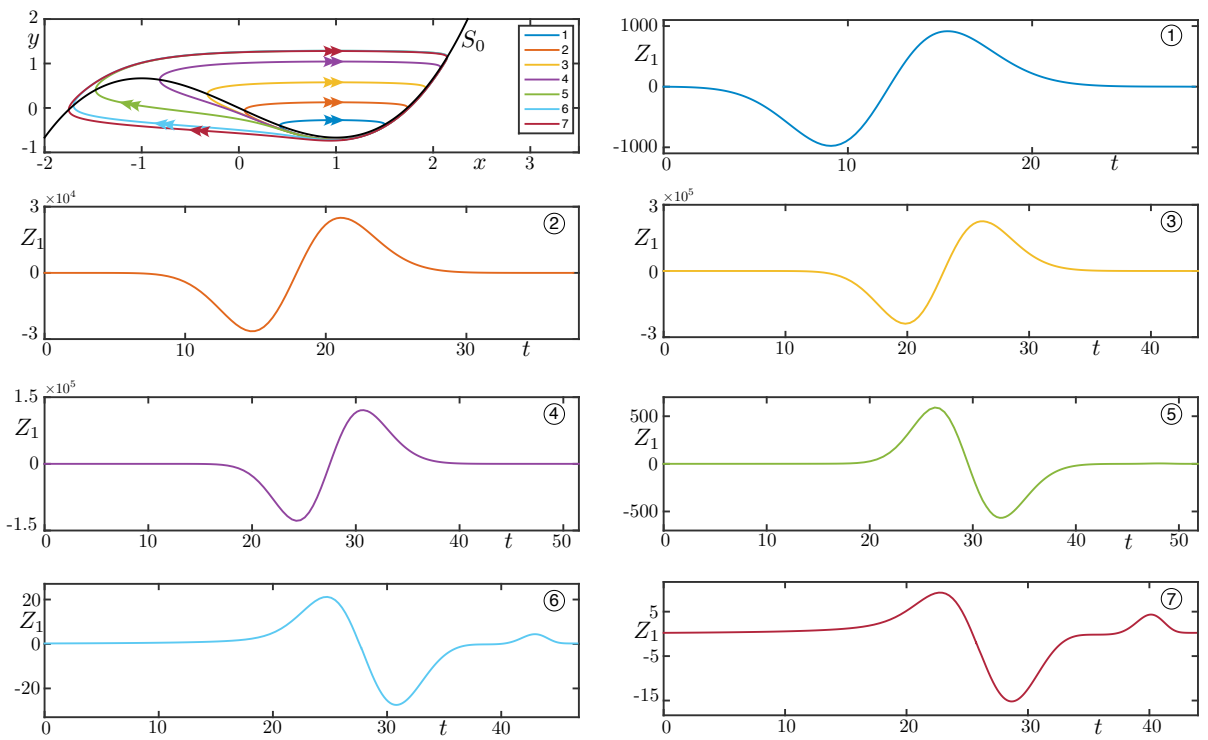

Figure 1: Top left panel: Canard orbits of the VDP system in the phase plane, for $\varepsilon=0.1$.

Panels 1-7: time profile of the first component of the adjoint solution associated with each canard cycle shown in the phase plane (together with the critical manifold $S_{0}:=\{y=f(x)\}$ ), keeping the same color coding. A qualitative change in the adjoint solution occurs in between Orbit 4 and Orbit 5, corresponding to the passage through the maximal canard cycle. 
with the adjoint equation. As hinted at above, the continuation procedure is divided into two steps.

In the first step, we initialize system (5) with the limit cycle $\gamma$ for the first two equations, and the trivial solution for the remaining two (which is trivially periodic). We need to obtain a non-trivial periodic solution of the adjoint equation which can be found by continuing system (5) in an extra parameter. Indeed, given that the trivial solution to the adjoint equation exists for all values of parameters $c$ and $\varepsilon$, by continuing in any of these we can only hope to find a branch point and switch at this bifurcation to the non-trivial solution branch. An alternative is to introduce a dummy parameter $\mu$, such that system (5) becomes

$$
\begin{aligned}
& x^{\prime}=y-f(x) \\
& y^{\prime}=\varepsilon(c-x) \\
& z_{1}^{\prime}=f^{\prime}\left(\gamma_{1}(t)\right) z_{1}+\varepsilon z_{2} \\
& z_{2}^{\prime}=-z_{1}+\mu,
\end{aligned}
$$

and to continue the starting solution in $\mu$ along a very small interval, as small as possible. It turns out that we can compute a branch in $\mu$ and stop at $\mu=10^{-8}$, which is indeed very small but sufficient to find a non-trivial solution of the extended problem (6).

180 Given that $\mu$ is very small, we can, in the second step, impose back $\mu=0$ and run a simple Newton iteration so as to converge to a non-trivial solution of the original extended problem (5). The advantage of using numerical continuation to compute a non-trivial solution to the adjoint equation along a canard cycle is that we can then continue the extended problem (5) in parameter $c$ and follow both the cycle and the associated periodic solution of the adjoint equation along the entire canard explosion.

Finally, the normalization condition (3) is required to close to the linear problem corresponding to the adjoint equation. Implementing this condition as part of our numerical continuation procedure can be a little delicate for small values of ${ }_{190} \varepsilon$, therefore we decided to use a periodic continuation in AUTO and apply the 
scaling that corresponds to (3) as a post-processing step. Note that we refrain from computing the Floquet bundle to obtain the non-trivial solution of the adjoint equation for this numerical problem since we only need any non-trivial solution to the adjoint equation, which we can then normalize appropriately. Starting from the Hopf bifurcation and continuing all the way to the relaxation regime, we can therefore follow the canard cycles by varying $c$ together with their associated adjoint solutions. Figure 1 shows some of the orbits lying in the headless canard and in the canard with head regimes. We observe a qualitative change in the adjoint solution, where $\max (x(t))$ point on the periodic orbit corresponds to the zero phase $\phi=0$, as the limit cycle $\gamma$ passes through the maximal canard.

In order to see whether or not the transitions that we observe in coupled VDP oscillators are system dependent, we next compute adjoints solutions associated with canards in a planar reduction of the $\mathrm{HH}$ model.

\subsubsection{Adjoints of canard cycles in a reduced Hodgkin-Huxley model}

A reduction of the classical $\mathrm{HH}$ model to two variables was analyzed from the viewpoint of canard dynamics in 63 ; the planar system has the form

$$
\begin{aligned}
C \dot{V} & =I-\bar{g}_{N a}\left[m_{\infty}(V)\right]^{3}(0.8-n)\left(V-V_{N a}\right)-\bar{g}_{K} n^{4}\left(V-V_{K}\right)-g_{L}\left(V-V_{L}\right) \\
\dot{n} & =\alpha_{n}(V)(1-n)-\beta_{n}(V) n,
\end{aligned}
$$

where $\alpha_{n}(V)=(0.01(V+55)) /(1-\exp [-(V+55) / 10]), \quad \beta_{n}(V)=0.125 \exp [-(V+$ $65) / 80], \quad m_{\infty}(V)=\alpha_{m} /\left(\alpha_{m}+\beta_{m}\right)$ with $\alpha_{m}=(0.1(V+40)) /(1-\exp [-(V+$ 40)/10]), $\left.\quad \beta_{m}=0.4 \exp [-(V+65) / 18]\right)$. Moehlis has shown in 63] that system (7) displays a canard explosion when parameter $I$ is varied, for the following fixed values of the other parameters: $\bar{g}_{N a}=120, \bar{g}_{K}=36, \bar{g}_{L}=0.3$, $V_{N a}=50, V_{K}=-77, V_{L}=-54.4, C=1$. After verifying numerically that the dynamics of $\mathrm{V}$ are much faster than the dynamics of $n$ and, hence, that the system effectively displays slow-fast dynamics, a formal asymptotic analysis was performed in $\varepsilon$ which appeared in the rescaled form of the slow equation 

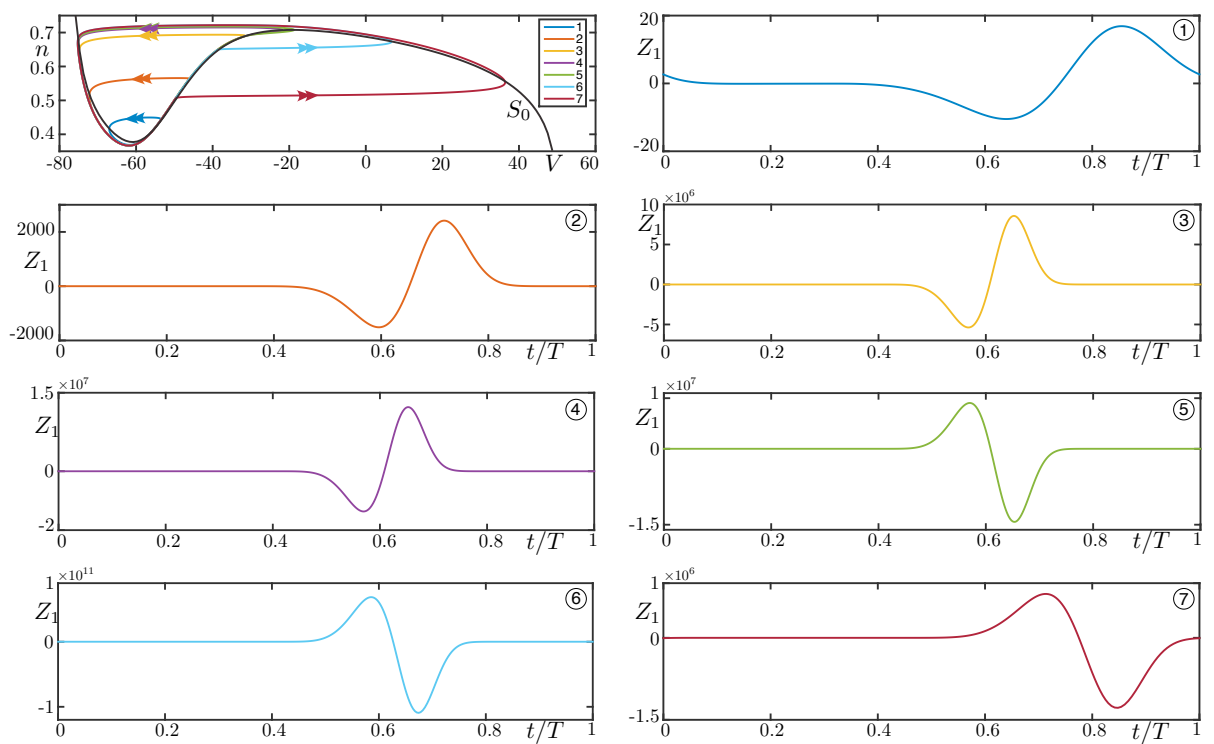

Figure 2: Top left panel: Canard orbits of the reduced HH system in the phase plane. Panels 1-7: time profile of the first component of the adjoint solution associated with each canard cycle shown in the phase plane (together with the critical manifold $S_{0}:=\{\dot{V}=0\}$ ), keeping the same color coding. A qualitative change in the adjoint solution occurs in between Orbit 4 and Orbit 5, corresponding to the passage through the maximal canard cycle. 
$\left(\dot{n}=\varepsilon\left(\alpha_{n}(V)(1-n)-\beta_{n}(V) n\right)\right)$ in 63 . Following the treatment of $\varepsilon$ as a small parameter in asymptotic analysis and obtaining an $\varepsilon$-expansion of the $I$-value at which the canard explosion occurs, $\varepsilon=1$ was plugged in the final formula.

Despite the instability of part of the canard branch in system (7), the continuation strategy allows to find solutions to adjoint equations. Since we are interested in the shape of the adjoints of the canard cycles lying on different sides of the repelling slow manifold, we can ignore the stability issue. Following the same continuation procedure described above, we compute adjoints of the 2D reduced HH system. Canard cycles and corresponding adjoint solutions are visualized in Figure 2, As in the VDP system, the transition from headless canards to canards with head changes qualitatively the adjoint solution.

\subsection{Consequences of a non-monotonic period function on the iPRC}

As shown in Figure 3 , the period function is non-monotonic along the canard explosion. It increases in the headless canard regime, reaches its maximum at the maximal canard and then decreases in the canard-with-head regime. The non-monotonicity of the period function along the explosive branch of canard cycles is one key aspect of the canard phenomenon in VDP-type systems, and the maximum of the period function can be used to detect numerically the maximal canard 64. The shape of this period function is sufficient to understand the effect of a perturbation of a canard cycle close enough to the lower fold of the critical manifold $S_{0}$. Indeed, $O(1)$ away from this fold point, a sufficiently small perturbation from the slow manifold takes the perturbed trajectory back to it very rapidly and therefore the effect of this perturbation is largely attenuated. This justifies that the solution to the adjoint equation along a canard cycle is close to zero for most of the cycle apart from the time interval corresponding to when the cycle is close to the lower fold (where the canard point is). On the other hand, near the lower fold of the critical manifold, the attraction to the slow manifold associated to the chosen canard cycle is weaker and the effect of the perturbation becomes large; see Figure 4(a2),(b2) for an illustration of this point. This effect can be understood by invoking the period function of the 
(a)

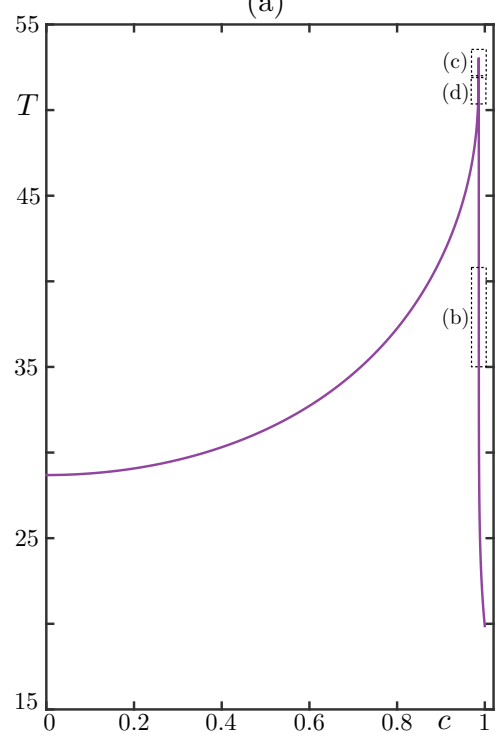

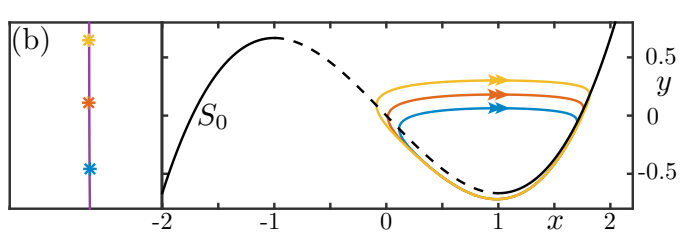
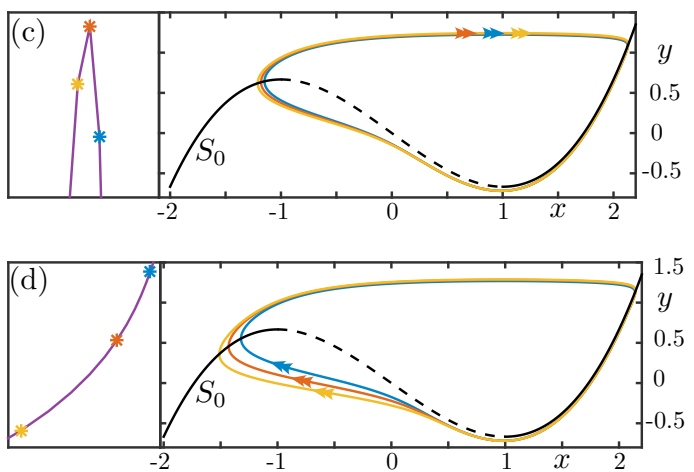

Figure 3: (a) Period of limit cycles along the canard explosion in the VDP system for $\varepsilon=0.1$; the parameter that varies is $c$. The period is increasing along the headless canard part of the branch, it reaches its maximum at the maximal canard and then decreases along the canard-with-head cycles. (b) Three headless canard cycles and their periods marked on the period curve. Smaller cycles have smaller periods. (c) Three cycles in the neighborhood of the maximal canard, together with their periods marked on the period curve. Canards with head and headless canards have very close periods in this vicinity. (d) Three canards with head and their periods marked on the period curve. Larger cycles have smaller periods. Also shown on panels (b) to (d) is the critical manifold $S_{0}$, on which solid (resp. dashed) parts represent stable (resp. unstable) branches. 
(a1)

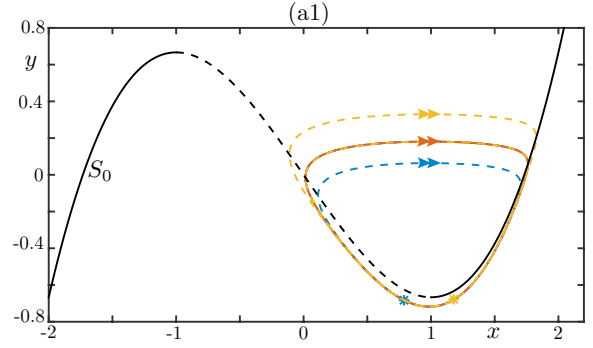

(b1)

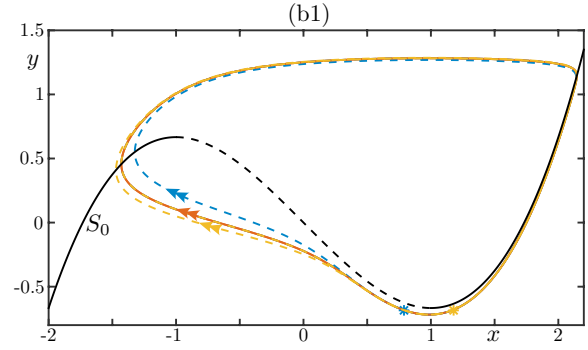

(a2)

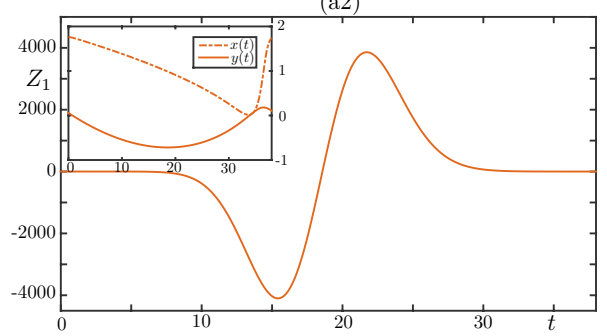

(b2)

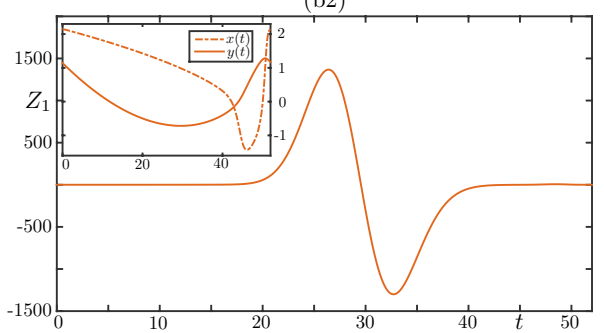

Figure 4: (a1, b1) Transient effect (dashed curves) of a small perturbation of the canard cycles (red solid curves) in the positive $x$-direction. (a2)-(b2) time profile of the first component of the adjoint solution associated with the red canard cycles and (inset) $(x(t), y(t))$ during one cycle. Perturbing a headless canard (resp. a canard with head) away from the attracting slow manifold (yellow asterisk) delays (resp. advances) its phase by driving it to a larger yet slower (resp. faster) yellow dashed cycle. Perturbing a headless canard (resp. a canard with head) towards the repelling slow manifold (blue asterisk) advances (resp. delays) its phase by driving it to a smaller yet faster (resp. slower) blue dashed cycle. 
branch of canard cycles.

First, consider headless canard cycles as represented in Figure 3(b). If we denote the period of the red cycle by $T_{\text {red }}$, then smaller canard cycles than the red one, like the blue cycle, have smaller periods whereas larger headless canard cycles, like the yellow one, have greater periods. Hence we have: $T_{b l u e}<$ ${ }_{250} T_{\text {red }}<T_{\text {yellow }}$. Therefore, an infinitesimal kick in the positive $x$ direction applied on the slow attracting segment of the red headless canard cycle near the fold (yellow dot in Figure 4(a1)) has the effect that the perturbed trajectory follows transiently a larger headless canard cycle (like the yellow one) before converging back to the red cycle. Given that the yellow cycle has a larger period, Applying such a kick on the slow repelling side of the red headless canard (blue dot in Figure 4(a1)) has the opposite effect given that in this case the perturbed trajectory first follows a smaller canard and, hence, has an advanced phase compared to the unperturbed one. Consequently, this qualitative argument justifies the sign of the adjoint solution along a headless canard cycle as shown in Figure 4(a2). As it can be observed from the $(x(t), y(t))$ flow shown in the inset of Figure 4(a2), the negative part of the first component of the adjoint $Z_{1}(t)$, which indicates phase delay, corresponds to the flow towards the fold. The sign of $Z_{1}(t)$ changes at the fold $(x=1)$ and then becomes positive as $(x(t), y(t))$ continue along the repelling branch. The situation for canards with head is entirely reversed: the period function is decreasing along the family of canardswith-head, hence three canards-with-head as shown in Figure 3(d) (blue, red, and yellow) have their periods satisfying the inequalities $T_{\text {blue }}>T_{\text {red }}>T_{\text {yellow }}$. Consequently, a similar phase plane argument as given above justifies that an infinitesimal kick on a canard with head on its slow attracting segment near the fold leads to a phase advance of the perturbed trajectory, whereas on the slow repelling segment it leads to a phase delay. This agrees with the adjoint solution computed along a canard with head and plotted in Figure 4(b2). Solution (x(t), $\mathrm{y}(\mathrm{t}))$ given the inner panel of Figure 4(b2) confirms that, indeed, $Z_{1}(t)$ takes 275 positive values along the flow towards the fold, changes its sign at the fold 
(a)

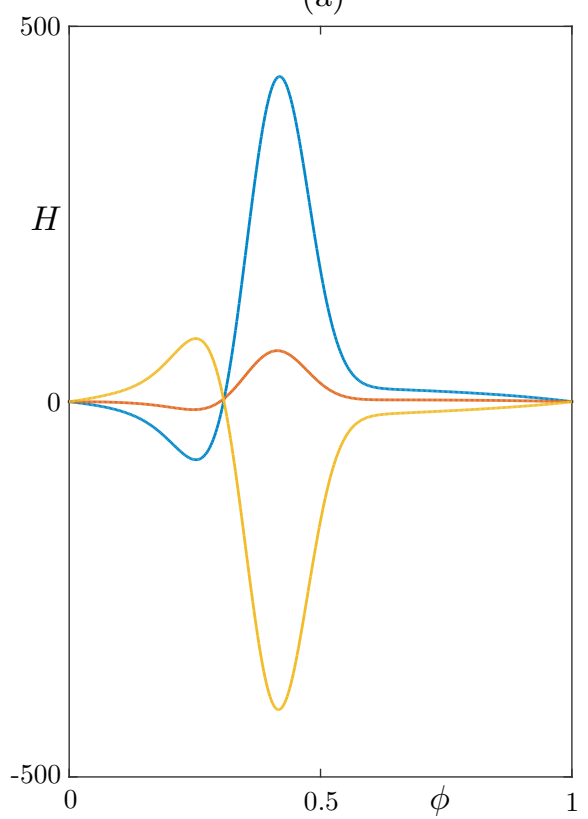

(b)

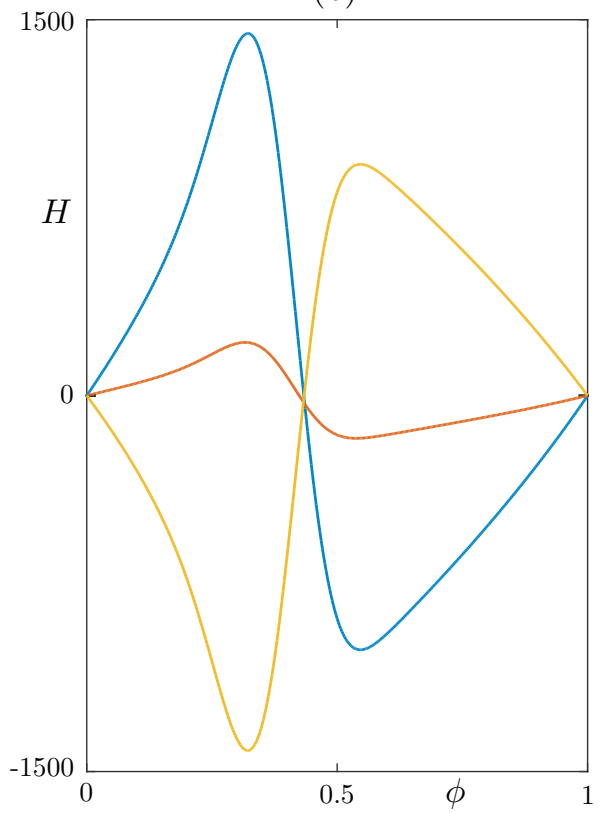

Figure 5: Interaction functions $H$ in the maximal canard neighborhood given in Figure 3 (c) for FF (panel (a)) and FS (panel (b)) coupling functions. The properties of $H$ reflect what is found for the solutions of the adjoint equation, i.e. the transition occurs in the neighborhood of the maximal canard.

$(x=1)$, then becomes negative as the solution $(x(t), y(t))$ moves away from the fold region. Note that invoking the period function to explain a change of shape and sign of the adjoint solution has been used in [55] in the context of so-called escape-release mechanism for the synchronization of half-center oscillators. Here we show that it also applies in the context of coupled canard oscillators.

\section{Synchronization properties of weakly coupled canard oscillators}

The behavior of the adjoint solutions (or equivalently, of the iPRCs) provides predictions on the collective behavior in the weak coupling regime via the interaction function which is the convolution of adjoint solutions and the coupling function [57, 7, 8, 5, 4. In coupled identical systems the interaction function of 
each oscillator reads:

$$
H\left(\phi_{j}-\phi_{i}\right)=\frac{1}{T} \int_{0}^{T} Z(t) U_{j}\left(\gamma(t), \gamma\left(t+\phi_{j}-\phi_{i}\right)\right) d t .
$$

where $\phi_{j}-\phi_{i}(i=\{1,2\}, j=3-i)$ is the phase difference between the two oscillators and $U$ is the coupling function. The dynamics of the phase difference, $\phi=\phi_{j}-\phi_{i}$, is described by the following equation

$$
\frac{d \phi}{d t}=\alpha[H(-\phi)-H(\phi)]=\alpha G(\phi) .
$$

where $1 \ll \alpha>0$ is the coupling strength. Equation $(9)$ has a stable solution at $\phi^{*}$ if $G^{\prime}\left(\phi^{*}\right)<0$, meaning that the two oscillators will synchronize with a phase difference $\phi^{*}$. The solution $\phi^{*}=0$ corresponds to IP synchronization, $\phi^{*}=\pi$ (or equivalently $\phi^{*}=0.5$ if the phase is rescaled to $\left.[0,1]\right)$ to AP synchronization, and any other values of $\phi^{*}$ corresponds to OP synchronization of coupled oscillators. In the case of coupled identical oscillators, that both IP and AP solutions are guaranteed to exist 65].

IP synchronization of two identical relaxation cycles (coming from oscillators with cubic-shaped fast nullclines) that are weakly coupled via fast to fast (FF) connections has been shown in [66, 29, 53, 30, 67, 27, outside the canard regime. In addition to FF coupling - which is the coupling function generally considered since it acts as a prototype for the electrical interaction between neuronal systems - we consider fast to slow (FS) coupling, which is not physiologically realistic but provides insight into understanding the interactions between perturbation and canards. The FF-coupled VDP oscillators read:

$$
\begin{aligned}
\varepsilon \dot{x_{i}} & =y_{i}+x_{i}-\frac{x_{i}^{3}}{3}+\alpha\left(x_{j}-x_{i}\right), \\
\dot{y}_{i} & =\left(c-x_{i}\right),
\end{aligned}
$$

and the FS-coupled system is given by

$$
\begin{aligned}
\varepsilon \dot{x_{i}} & =y_{i}+x_{i}-\frac{x_{i}^{3}}{3}, \\
\dot{y}_{i} & =c-x_{i}+\alpha\left(x_{i}-x_{j}\right) .
\end{aligned}
$$



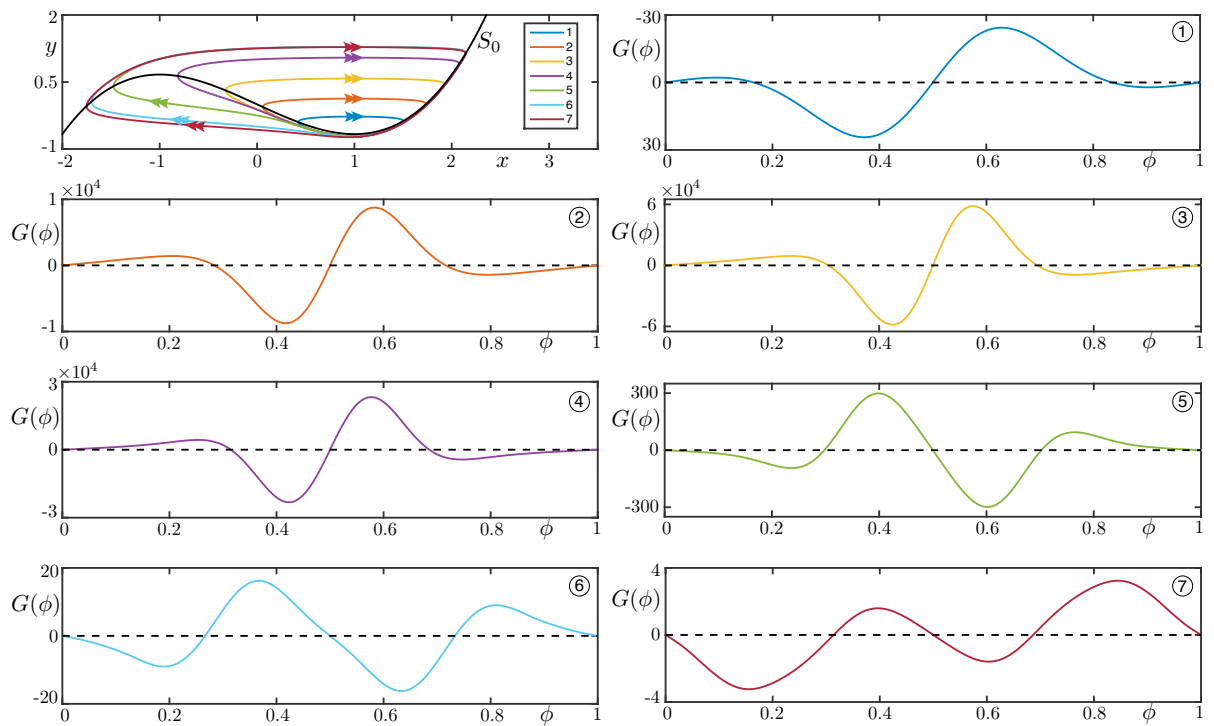

Figure 6: Selection of canard cycles of the VDP oscillator in the phase plane $(x, y)$ (top left panel) together with the corresponding $G$ functions (panels 1 to 7 ; the phase $\phi$ is rescaled to $[0,1])$.

The effect of a small perturbation on the canard cycles in the neighborhood of the lower fold of the critical manifold $S_{0}$, is different for canards with head than for headless canard cycles, as revealed by the corresponding adjoint solutions; see Figure 1. This qualitative change occurs at the maximal canard. Figure 5 shows the interaction functions $H$ associated with the cycles in the neighborhood of the maximal canard (shown in Figure 3 (c)) interacting via FF (panel (a)) and FS (panel (b)) connections. Given that a headless canard cycle resembles more the maximal canard (the maximal canard being a maximal headless canard), the amplitude of the corresponding function $H$ decreases while the number of zeros, that is, solutions to $H\left(\phi^{*}\right)=0$, and the sign of $H^{\prime}\left(\phi^{*}\right)$ remain the same. The sign of $H^{\prime}\left(\phi^{*}\right)$ changes when the cycle moves to the canard-with-head regime, while the number of zeros $\phi^{*}$ is preserved.

The function $G$ (see Figure 6) is computed for the cycles (whose adjoints 310 are presented in Figure 1) interacting via FF coupling. The location of the zeros $\phi^{*}$ of $G$, and the sign of its derivative at such points, determine the type 
and stability of synchronized state of the coupled system. The IP synchronized solution which exists for the Hopf cycles (not shown on this figure) loses stability along the canard explosion (due to the high sensitivity to perturbation resulting from the passage near the fold of the critical manifold $S^{0}$ ) and a stable OP solution appears for the headless canard cycles (orbits 1-4). The phase difference of the stable OP solution increases as the cycle approaches the maximal canard (Panels 1-4). Bistability appears for the canards-with-head (orbits 5-7), where IP and AP solutions are the stable synchronous solutions and the $\mathrm{OP}$ is the unstable solution (Panels 5-7).

The information obtained with the function $G$ about synchronized states of the weakly coupled VDP system with FF coupling, can be confirmed by a numerical bifurcation analysis of the coupled system in question. We have performed this analysis by continuing synchronous states of system (10) (including the ones which are not visualized in Figure 60 in parameter $c$. The result is presented in Figure 7 where the chosen solution measure is the difference between the $x$-component of each oscillator at time $t=0, x_{2}(0)-x_{1}(0)$, regardless of its varying amplitude as a function of $c$. That measure has the same interpretation as the phase difference for these simple orbits and it is often used in the analysis of weakly coupled oscillators [2. Panels (b) to (d) are successive zooms of panel (a) in the region corresponding to maximal canards for each oscillator. The properties of the synchronized states of the FF-coupled cycles are tracked starting from the double Hopf bifurcation point at $c=c_{H o p f}=1$ down to the relaxation regime near $c \approx 0.615$. We consider a fixed coupling strength $\alpha=10^{-5}$ for which the weakly coupled oscillators theory is expected to be valid; a detailed discussion on the effect of $\alpha$ is presented in Section 4 One stable and two (symmetric) unstable branches, which correspond to IP and AP solutions, respectively, appear at $c=c_{H o p f}$. The IP solution undergoes a pitchfork bifurcation through which it loses its stability as a stable OP solution appears (Panel (b)). The OP branches become unstable at a PD bifurcation which is followed by a PD cascade corresponding to $2 n T$-periodic stable synchronous solutions (Panel $(\mathrm{d})$ ), where the interaction function analysis is not 
(a)

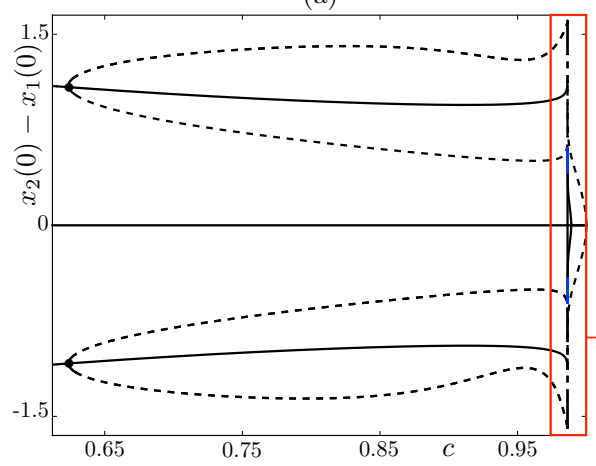

(c)

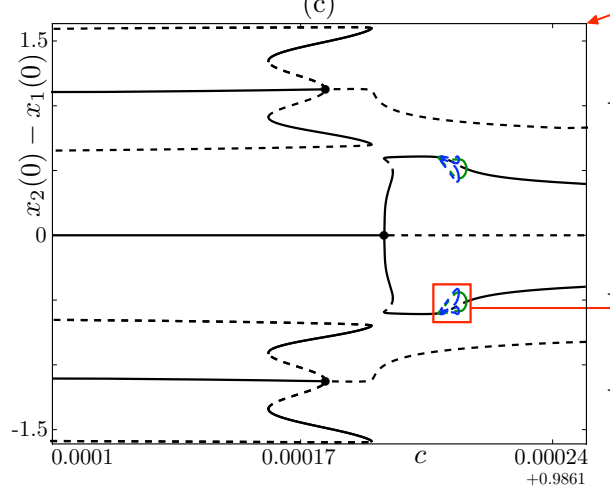

(b)

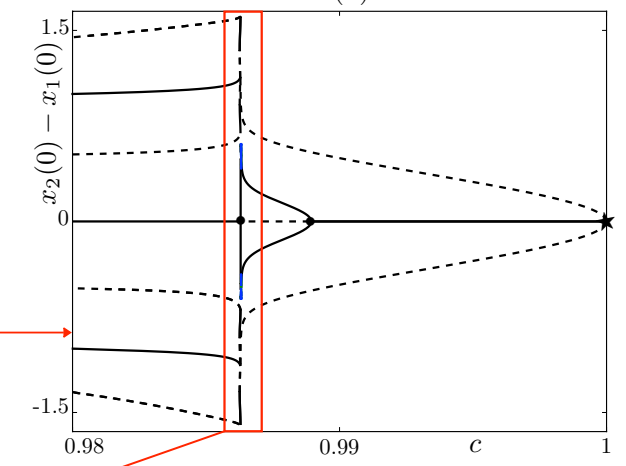

(d)

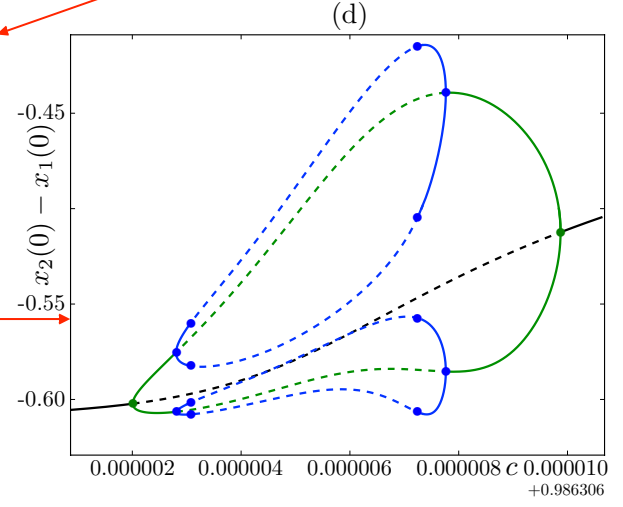

Figure 7: Bifurcation diagram of system 10 with respect to variations of $c$ for $\alpha=10^{-5}$, from the Hopf regime to the relaxation regime. The output solution measure is the difference between the first components of each oscillator at time $t=0$. The region of the maximal canard is enlarged from left to right and top to bottom panels. Black dots in panels (a) to (c) denote pitchfork bifurcation points; the black star in panel (b) corresponds to the double Hopf point that initiate the periodic regime in this coupled system; colored dots in panel (d) denote PD bifurcation points. 
(a)

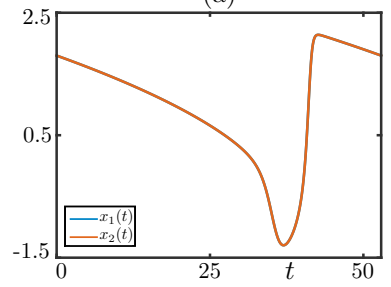

(d)

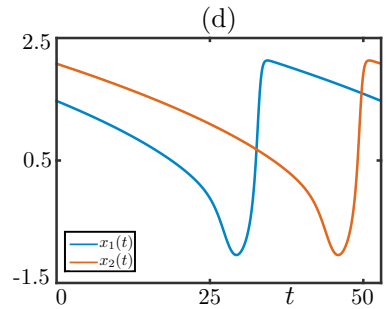

(b)

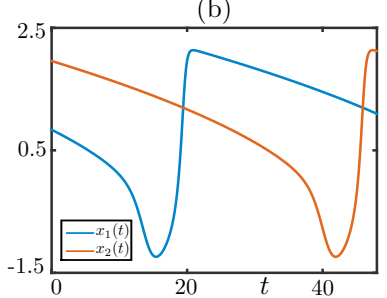

(e)

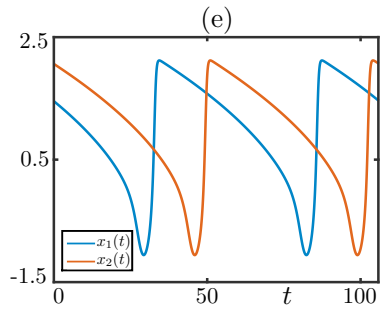

(c)

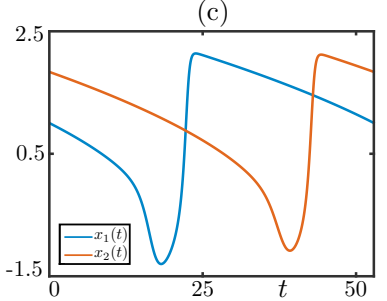

(f)

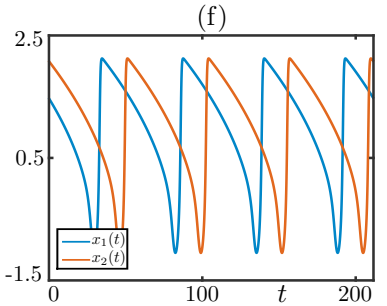

Figure 8: Coexisting stable IP (a), AP (b) OP (c) solutions for $c=0.986267$ from Figure 7 (c). Stable $T$-periodic solution for $c=0.98631587277$ (d), $2 T$-periodic solution for $c=0.9863137635$ (e), and $4 T$-periodic solution for $c=0.98631334783$ (f), from Figure 7 (d).

valid. The $T$-periodic OP branches become stable again via a second PD bifurcation. It changes its stability two times via a couple of fold bifurcations before connecting to the second pitchfork bifurcation point on the IP branch that restabilizes the IP state.

The unstable AP branch that appears at $c_{\text {Hopf }}$ becomes stable at the maximal canard of the coupled system through a pitchfork bifurcation (Panel (c)). The stable AP and OP solution related to this pitchfork bifurcation coexist with stable IP solutions for a some range of $c$ in the neighborhood of the maximal canard. For smaller values of $c$, IP and AP remain stable, while OP states are unstable.

The bistability regions (illustrated in Figure 8) already hinted at with the investigation of the function $G$, are well identified through the continuation analysis, in particular the coexisting stable IP and stable AP states (canards with head and relaxation cycles) born near the maximal canard solutions. This intricate bifurcation structure unveils a main connection between the stable IP and the AP states through the double Hopf point at $c=c_{H o p f}$, which gives 
rise to both the IP stable state and a branch of unstable AP states. Decreasthis aspect in the next section.

\section{Effect of the coupling strength $\alpha$}

The interaction function analysis reveals the existence and stability of synchronous states for weakly coupled oscillators, although how "weak" the cou-

pling should be in order that the theory applies is questionable. For instance, it was shown in [14] that for leakly integrate-and-fire type of oscillators the $H$ function analysis is valid for moderate coupling strengths, whereas other papers 
(see e.g. [68, 69]) have mentioned a loss of 1:1 phase locking estimated by the interaction function analysis. In the case of coupled canard-explosive systems where the properties of the underlying oscillators vary brutally in parameter ranges that are exponentially small in time-scale parameter $\varepsilon$, the notion of weak coupling can be even more vague. For instance the region with cascades of PD bifurcations, highlighted in Figure 7(d) and corresponding to cycles that are close to the maximal canard regime (under weak coupling of strength $\alpha=10^{-5}$ ), gives a good numerical evidence that canard orbits are very sensitive to perturbations and that the validity of the interaction function analysis is limited in such cases.

In order to investigate this aspect further, we next consider the phase difference dynamics of two coupled identical headless canard cycles for a $c$-value in the neighborhood of the maximal canard, as a function of the coupling strength $\alpha>0$. This numerical continuation study will focus both FF and FS interactions. The aim is to identify what range of the perturbation strength can give rise to interesting canard-mediated dynamics that are not predicted by the interaction function analysis but whose existence can be justified using slow-fast arguments.

Fast-to-Fast (FF) coupling. The bifurcation structure in $\alpha$ for this case in presented in Figures 9 and 10 (zoomed views); associated solution profiles are shown in Figures 11 and 12. A stable OP synchronous state with a phase difference $\phi^{*}=0.34$ is predicted by the interaction function analysis for the case of two headless canard cycles with FF-coupling, that is, for system 110 ; see Figure 5 (a) and Figure 6 panel 4. Using the bifurcation diagram presented in Figure 9, we can conclude that this $\mathrm{OP}$ regime persists for $\alpha \in\left(0,6 \cdot 63371 \times 10^{-5}\right]$. It loses its stability at $\alpha \approx 6.63371 \times 10^{-5}$ via a $\mathrm{PD}$ bifurcation where the interaction function result is violated, and consequently, not valid for greater coupling strengths. Switching branch at this PD point reveals the presence of a PD cascade, for which we compute only a few subsequent branches. Among the stable part of these branches of period-2nT synchronous solutions (near which chaotic orbits 


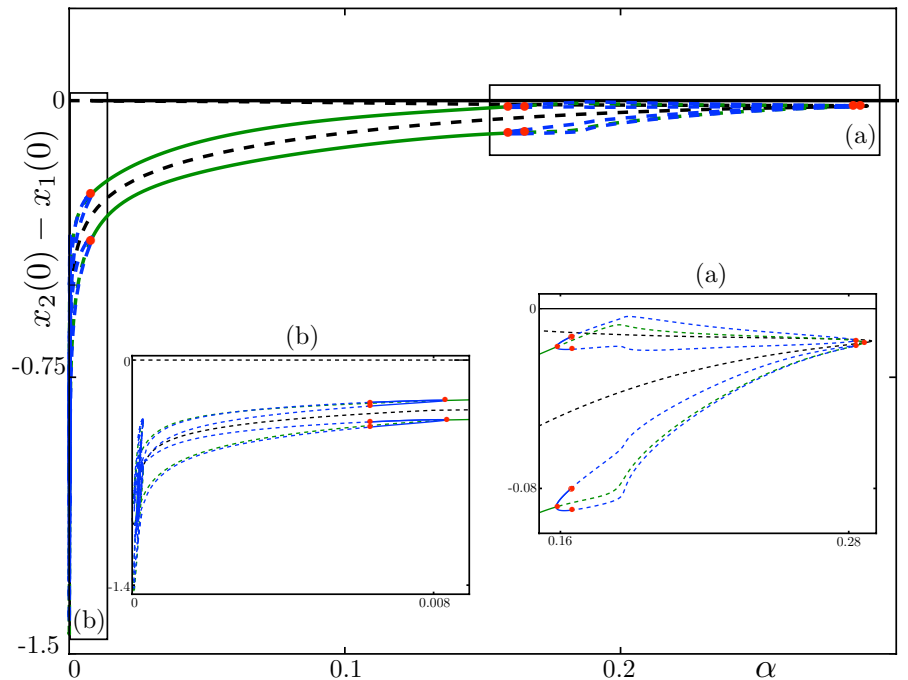

Figure 9: Continuation in $\alpha$ for the FF-coupled VDP system for a $c$ value in the vicinity of the maximal canard. Inset panels (a) and (b) are zoomed viewed of different parts of the main panel. Bifurcation points (mainly PD bifurcations) are indicated by red dots. T-periodic (black), $2 T$-periodic (green) and $4 T$-periodic (blue) branches coexist with stable (solid) and unstable (dashed) solutions.

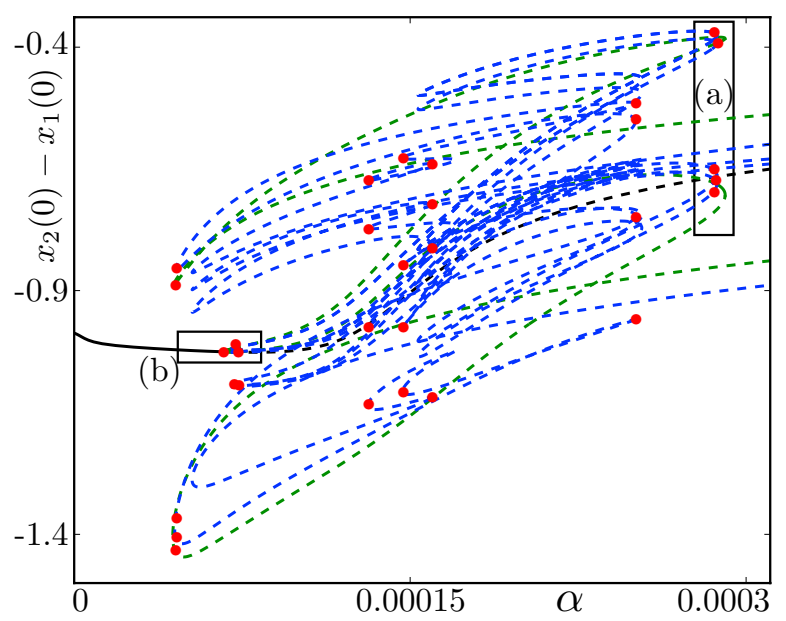

(a)

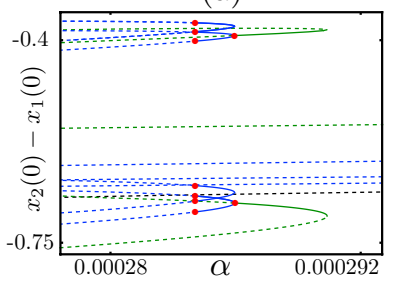

(b)

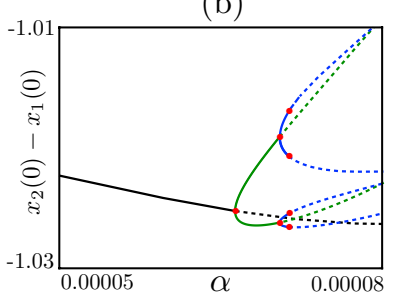

Figure 10: Continuation in $\alpha$ for the FF-coupled VDP system in the maximal canard regime: zoomed view from Figure 9 in the region of PD cascades (most of the computed PD bifurcation points being highlighted by colored dots). 
(a1)
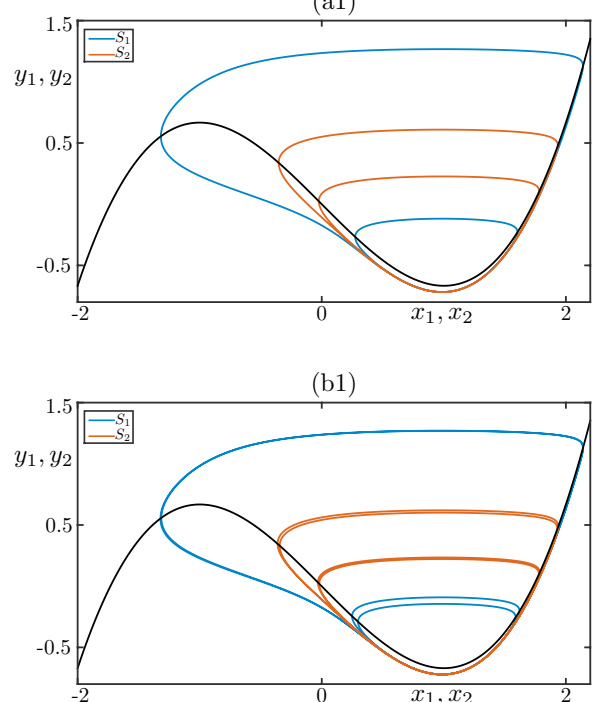

(a2)

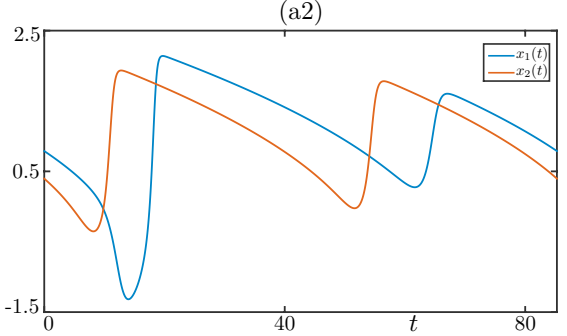

(b2)

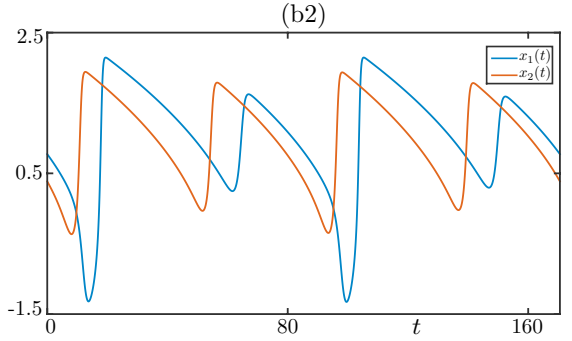

Figure 11: Period 2T (top panels) and period $4 T$ (bottom panels) non-identical OP synchronous states for the FF-coupled system in the maximal canard regime, illustrating the spike suppression scenario. Values of the coupling strength $\alpha$ are $2.86959 \times 10^{-4}$ in panels (a1)-(a2) and $2.85359 \times 10^{-4}$ in panels (b1)-(b2).

surely exist too), that is, for a coupling strength $\alpha \in\left(6.63371 \times 10^{-5}, 0.0083195\right]$, there exists a family of solutions displaying what we call "spike suppression". This scenario corresponds to when one of the oscillators spikes by following a canard with head while the other always remain in the headless canard regime. Regarding the IP solution branch, it becomes stable at $\alpha \approx 0.0085633416545$ and coexists, for $\alpha \in[0.0085633416545,0.289498]$, with the $2 n T$-periodic headless canard solution branch.

Fast-to-Slow (FS) coupling. The bifurcation structure in $\alpha$ for this case in presented in Figure 13. associated solution profiles are shown in Figure 14. The stable IP synchronization state predicted by the interaction function analysis for the FS-coupling (Figure 5 (b)) becomes unstable at $\alpha \approx 0.007498445$ (Figure 13 (a)) via a subcritical PD bifurcation that introduces an unstable $2 T$-periodic branch which becomes stable at $\alpha \approx 8.74785268 \times 10^{-5}$, where the interaction function analysis loses its validity. Continuing that branch leads to the detec- 
(a1)

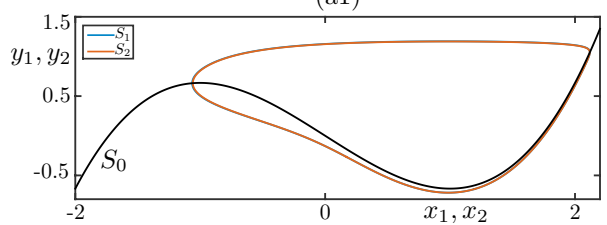

(b1)

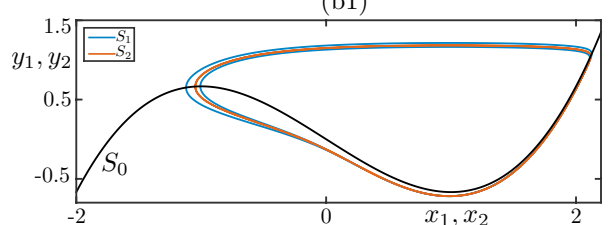

(c1)

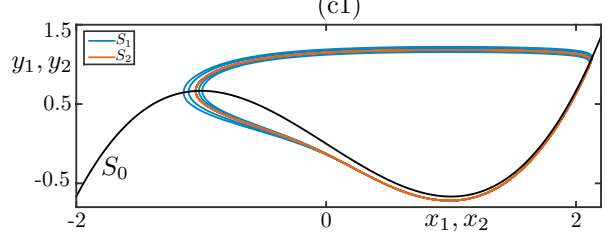

$(\mathrm{a} 2)$

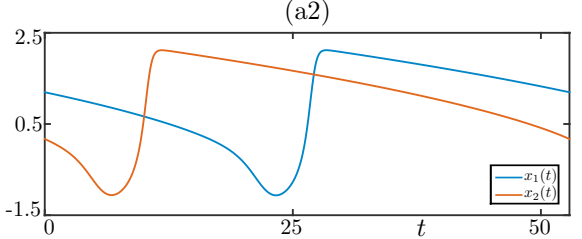

(b2)

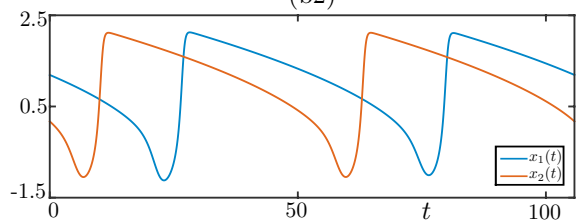

(c2)

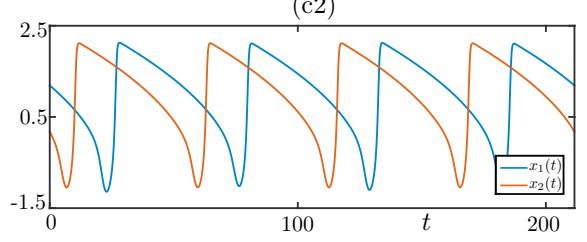

Figure 12: Period T (top panels, identical), period 2T (middle panels, non-identical) and period $4 T$ (bottom panels, non-identical) stable OP synchronous states of the FF-coupled VDP system in the maximal canard regime. The phase differences for these states are coherent with the interaction function analysis. Values of the coupling strength $\alpha$ are $6.63371 \times 10^{-5}$ in panels (a1)-(a2), $7.04717 \times 10^{-5}$ in panels (b1)-(b2) and $7.13322 \times 10^{-5}$ in panels (c1)-(c2). Left panels: Trajectories projected onto the $\left(x_{i}, y_{i}\right)$ planes. Right panels: Time series of the $x_{i}$ coordinates.

tion of further PD bifurcations organized in a cascade, which we compute only the beginning of; see Figure 13 (b). These $n T$-periodic branches correspond to families of solutions displaying what we call "spike alternation", that is, a scenario for which both oscillators of the FS-coupled system follow subsequently a headless canard segment and then a canard-with-head segment, hence performing an MMO [40; see Figure 14 for an illustration on such MMO cycles with period $T, 2 T$ and $4 T$ on (a), (b) and (c) panels, respectively. Depending on the value of the coupling strength $\alpha$, the oscillators may follow the same or different canard trajectories.

On both FF- and FS-coupled canard systems, we have observed using a numerical bifurcation analysis the proximity of several stable solution branches 
(a)

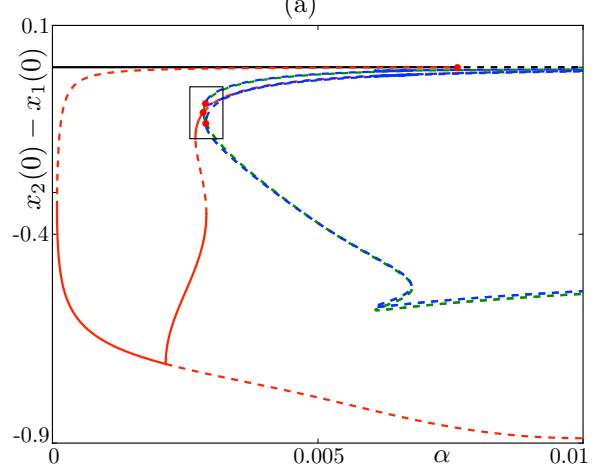

(b)

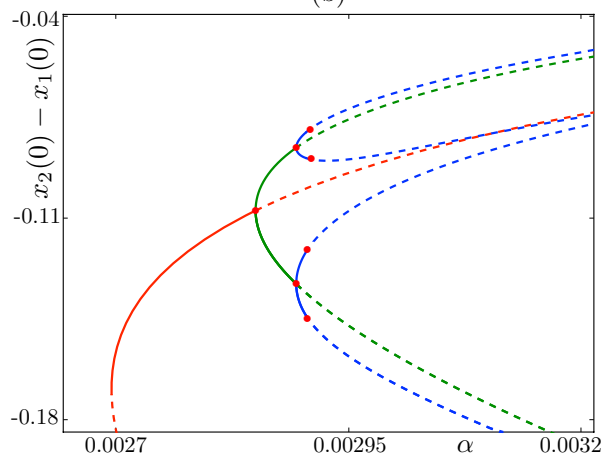

Figure 13: Continuation in $\alpha$ for the FS-coupled VDP system. Bifurcation points (PD bifurcations) are indicated by red dots. Both stable (solid) and unstable (dashed) parts of $T$-periodic (black), 2T-periodic (red), 4T-periodic (green) and $8 T$-periodic (blue) branches are shown.

(a1)

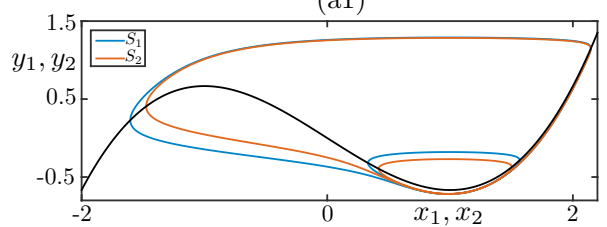

(b1)

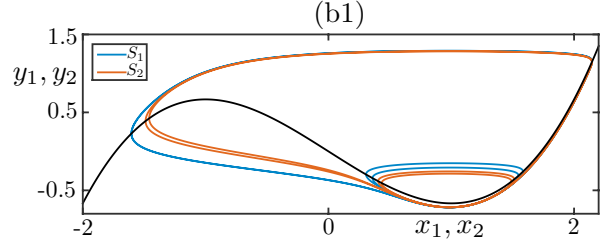

(c1)

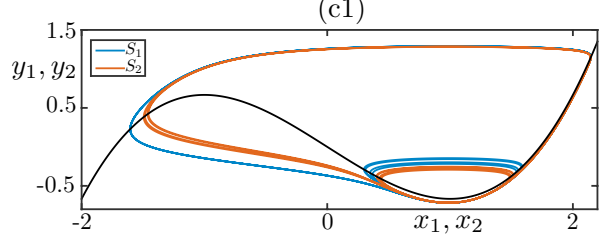

(a2)

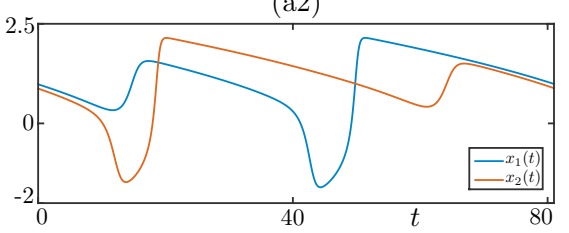

(b2)

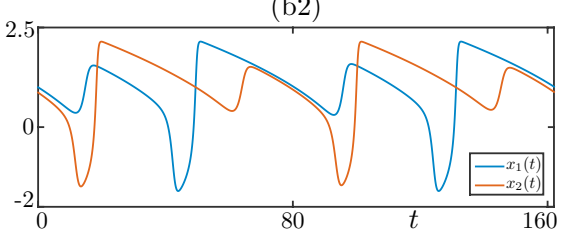

(c2)

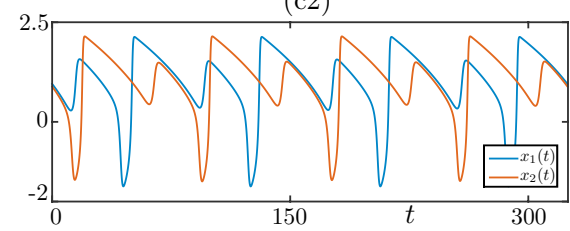

Figure 14: Period $T$ (top panels), $2 T$ (middle panels) and $4 T$ (bottom panels) stable nonidentical OP synchronous states displaying spike alternation for the FS-coupled system near the maximal canard regime. Values of the coupling strength $\alpha$ are $2.8502655978 \times 10^{-3}$ in panels (a1)-(a2), $2.8939484985 \times 10^{-3}$ in panels (b1)-(b2) and $2.9039987077 \times 10^{-3}$ in panels (c1)-(c2). Left panels: Trajectories projected onto the $\left(x_{i}, y_{i}\right)$ planes. Right panels: Time series of the $x_{i}$ coordinates. 
with complicated oscillatory patterns mixing passages along headless canards and along canards with head. In the context of neuronal systems, these solutions alternate subthreshold oscillations and spikes. These solutions are not predicted by the interaction function analysis typically employed in weakly coupled oscillator studies. However, one can justify their existence by invoking the presence in such systems of repelling (Fenichel) slow manifolds, which are known to be exponentially close to each other (in the timescale separation parameter $\varepsilon$ ). Therefore, the presence of these manifolds near the middle branch of the critical manifold $S_{0}$ of each individual slow-fast oscillator can allow to explain why, for values of the coupling strength $\alpha$ that are larger that exponentially small quantities, synchronized states of the coupled system may follow these manifolds on one side (subthreshold regime) or the other (spiking regime) while staying very close to the boundary (well approximated by maximal canards). In other words, when two identical slow-fast systems are weakly coupled, the existence of a repelling slow manifold and of an associated maximal canard trajectory in the uncoupled system can give rise to solutions to the full (coupled) system for which each node follows this maximal canard on opposite sides, hence, separate after an $O(1)$ time. This can happen as soon as the coupling is stronger than an exponentially small function of $\varepsilon$ and can therefore be responsible for the presence of canard-induced states in the coupled system; see [51] for an example of this phenomenon in weakly coupled folded-singularity systems.

\section{Discussion}

In this paper, we have extended previous results on weakly coupled slow-fast oscillators to the canard regime, both from theoretical and numerical perspectives. Our main finding is that the behavior of adjoint solutions (or equivalently, of iPRCs) changes qualitatively when the canard cycle under consideration is moving (as the canard parameter is varied) along the associated explosive branch. Indeed, the sign and shape of the adjoint solutions flip as the underlying canard cycle goes from the headless canard regime to the canard-with-head 
regime, the transition taking place at the maximal canard cycle, which in particular sheds new light onto the previously unnoticed role played by this special canard in the context of coupled slow-fast oscillators, deeply connected to the fact that it corresponds to a critical point of the period function 64. This change of behavior of adjoints of canard cycles upon infinitesimal perturbations can be explained by the peculiar known property of the period function of a canard-explosive branch, which can be summarized as follows: larger headless canards have greater periods, whereas larger canards-with-head have smaller periods. As explained in Section 3, this arguments is fully applicable when the perturbation is applied near the fold point of the critical manifold corresponding to the canard point, and its validity is weakened as the perturbation is applied further away from this fold point, where the contraction towards the unperturbed cycle rapidly annihilates the effect of the perturbation. This justifies that adjoints computed along canard cycles are very close to zero during most of the cycle except along a time interval corresponding to when the canard cycle passes near the fold (canard) point of the critical manifold $S_{0}$. Nevertheless, the explanation that we provide is valid for the most informative part of the adjoint solutions and bears consequences on the synchronized solutions of coupled canard systems.

We have shown this mechanism for a prototypical canard oscillator, namely the VDP system, but it is clearly applicable to all excitable systems of this form, in particular, to slow-fast type-II neuron models such as the reduced HH model studied in 63. This opens the way to a renewed understanding of iPRCs in such neuron models, from the Hopf cycles (whose adjoint solutions will qualitatively look like those associated with small headless canard cycles) to the spiking cycles (whose adjoint solutions will qualitatively look like those associated with canards with head). In particular, our findings can be related to recent work on isochrones since PRC analysis originates in the study of phase models and isochrones [70. Recently the isochrons of canard cycles were investigated numerically in [71, 72] where evidence was given that their properties change in the vicinity of the maximal canard neighborhood; this is likely to be closely 
linked with the results presented here. While a full comparison of these two aspects of canard cycles' phase properties goes beyond the scope of the present coupling strength $\alpha$. PD bifurcations and chaotic trajectories in VDP-like systems under periodic perturbation have been studied in e.g. [73, 16, 74. In the 
present study, we unveiled a complex web of period- $2 n T$ branches suggestive of the presence of nearby chaotic attractors, which we chose not to investigate. Instead, we highlighted these further synchronous states, all existing close to maximal canard solutions but not all predicted by standard interaction function analysis. Being close to a maximal canard, hence to threshold, these solutions may contain both passages near headless canards and near canards-with-head, therefore an alternation between subthreshold oscillations and spikes. Even when the classical weakly coupled theory may not apply, the slow-fast phase plane structure of the underlying single canard oscillator enables one to understand why such mixed-mode oscillatory synchronous states can arise for small to moderate coupling strength, owing to the geometry and proximity between 545 families of repelling slow manifolds. As a question for future work, we plan to investigate the relevance of these complicated synchronous states in the context of neuron models, where canards-with-head may be considered as not so rare events but rather as spikes with a slow activation.

Control aspects of canard cycles have been studied in [75] where the authors have obtained MMOs, cascades of PD bifurcations and chaotic behavior in a FHN-type relaxation oscillator depending on the control setup. Developing control strategies for reaching desired spiking behavior in coupled systems can be a future direction of our study.

Finally, as an appendix, we also provided an analytical formula for the adjoint solutions associated with limit cycles of Liénard systems, which gives reasonable numerical results for headless canard cycles.

This work is only a first step towards extending canard studies to the realm of weakly coupled oscillators and, more generally, to weakly connected networks. It is not rigorous yet but we have identified the main geometrical structures that play a pivotal role in shaping the main family of synchronous solutions to coupled planar slow-fast systems in the canard regime. Moreover, we have highlighted the central role of the maximal canard in organizing the synchronization properties of such systems. Maximal canards have been identified as the spiking threshold in excitable neuron models [76, 77]. Our study on weakly coupled pla- 
nar systems, which are reduced models for excitable neurons, has underlined the relation between synchronisation, excitability and maximal canard by relating it to the properties of the period function of the canard explosion. The interaction between the slow-fast structure and the weak coupling has given quite rich dynamics in these simple planar systems having already a two slow/two

fast structure under coupling. Similar possible links between synchronisation, excitability and maximal canards certainly deserves further attention in coupled slow-fast systems of higher dimensions as well as in larger networks. Beyond the effect of canard-explosive dynamics on synchronization, we plan in the near future to investigate similar effects in (at least three-dimensional) systems with canards organized by folded singularities [78] as well as in systems with slowly varying quantities, such as bursting systems where spike-adding canard explosions will be likely to have a dramatic effect on the synchronization properties of coupled bursters [79, 41].

\section{Appendix A. Analytical expression for adjoints of canard cycles}

580 Here we use classical results from the theory of linear differential equations [80] as well as unpublished results by Schecter [81] in order to derive an expression for the periodic solution of the adjoint problem associated with a limit cycle of a Liénard system. This extends the approach taken by Izhikevich in [53], who considered the case of relaxation cycles by taking the limit $\varepsilon=0$.

55 Izhikevich's formulation is not applicable to canard cycles due to the presence of the folds of the critical manifold $S_{0}$ which gives rise to canard dynamics and requires to have $\varepsilon \neq 0$ in the computation of the adjoints.

We consider the following VDP type slow-fast system written in Liénard form

$$
\begin{aligned}
& x^{\prime}=y-f(x):=F(x, y) \\
& y^{\prime}=\varepsilon(c-x):=\varepsilon G(x, y),
\end{aligned}
$$

where $f(x)=x^{3} / 3-x$ is a cubic function and the prime denotes differentiation with respect to the fast time $t$. We consider a canard cycle solution of 
system A.1, that is, a periodic solution $\gamma(t)=(x(\varepsilon t), y(\varepsilon t))$.

The linearized system associated with A.1 along $\gamma$ is given by

$$
\begin{aligned}
v^{\prime} & =-f^{\prime}\left(\gamma_{1}(t)\right) v+w \\
w^{\prime} & =-\varepsilon v
\end{aligned}
$$

which we can recast as a second-order linear differential equation

$$
v^{\prime \prime}(t)+f^{\prime}\left(\gamma_{1}(t)\right) v^{\prime}(t)+\left(f^{\prime \prime}\left(\gamma_{1}(t)\right)+\varepsilon\right) v(t)=0 .
$$

An obvious solution of A.3 is $\left(\gamma_{1}^{\prime}(t), \gamma_{2}^{\prime}(t)\right)$. Recall that if one knows a particular solution $y_{*}$ of the second-order linear differential

$$
y^{\prime \prime}(t)+p(t) y^{\prime}(t)+q(t) y(t)=0,
$$

then one can obtain another solution $y_{\#}$, non-proportional to the first one hence forming a basis of the space of solutions together with the first one using a variation of constant type formula, that is,

$$
y_{\#}(t)=u(t) y_{*}(t)
$$

with $u$ given in general integral form by

$$
u(t)=\int_{0}^{t} \frac{\exp \left(-\int_{0}^{s} p(\sigma) d \sigma\right)}{y^{2}(s)} d s .
$$

Therefore, knowing the solution $\left(\gamma_{1}^{\prime}(t), \gamma_{2}^{\prime}(t)\right)$ of the linearized system written as a second-order equation A.3, a non-proportional solution is given by $(v(t), w(t))$ with

$$
\begin{aligned}
& v(t)=u(t) \gamma_{1}^{\prime}(t)=\gamma_{1}^{\prime}(t) \int_{0}^{t} \frac{\exp \left(-\int_{0}^{s} f^{\prime}\left(\gamma_{1}(\sigma)\right) d \sigma\right)}{\gamma_{1}^{\prime 2}(s)} d s \\
& w(t)=v^{\prime}(t)+f^{\prime}\left(\gamma_{1}(t)\right) v(t)
\end{aligned}
$$

Hence we have

$$
\begin{aligned}
w(t) & =u^{\prime}(t) \gamma_{1}^{\prime}(t)+u(t)\left(\gamma_{1}^{\prime \prime}(t)+f^{\prime}\left(\gamma_{1}(t)\right) \gamma_{1}^{\prime}(t)\right) \\
& =\frac{\exp \left(-\int_{0}^{t} f^{\prime}\left(\gamma_{1}(s)\right) d s\right)}{\gamma_{1}^{\prime}(t)}+u(t) \gamma_{2}^{\prime}(t) .
\end{aligned}
$$


The adjoint equation associated with system A.1 along the limit cycle $\gamma$ is given by

$$
\dot{\mathrm{Z}}=-\mathrm{J}(\gamma(t))^{T} \mathrm{Z}
$$

where $\mathrm{Z}$ is a two-dimensional real vector and $\mathrm{J}(\gamma(t))$ is the Jacobian matrix evaluated along the solution $\gamma$. Following [81, we write the solution to equation A.7 in the form

$$
\mathrm{Z}^{T}(t)=\exp \left(\int_{0}^{t} f^{\prime}\left(\gamma_{1}(s)\right) d s\right)\left[\begin{array}{ll}
-s_{2} & s_{1}
\end{array}\right],
$$

600 $\alpha$ as a function of $\beta$ :

$$
\alpha=\frac{-\beta \exp \left(\int_{0}^{T} f^{\prime}\left(\gamma_{1}(s)\right) d s\right) \int_{0}^{T} \frac{\exp \left(-\int_{0}^{s} f^{\prime}\left(\gamma_{1}(\sigma)\right) d \sigma\right)}{\gamma_{1}^{\prime 2}(s)} d s}{\exp \left(\int_{0}^{T} p(s) d s\right)-1}(
$$



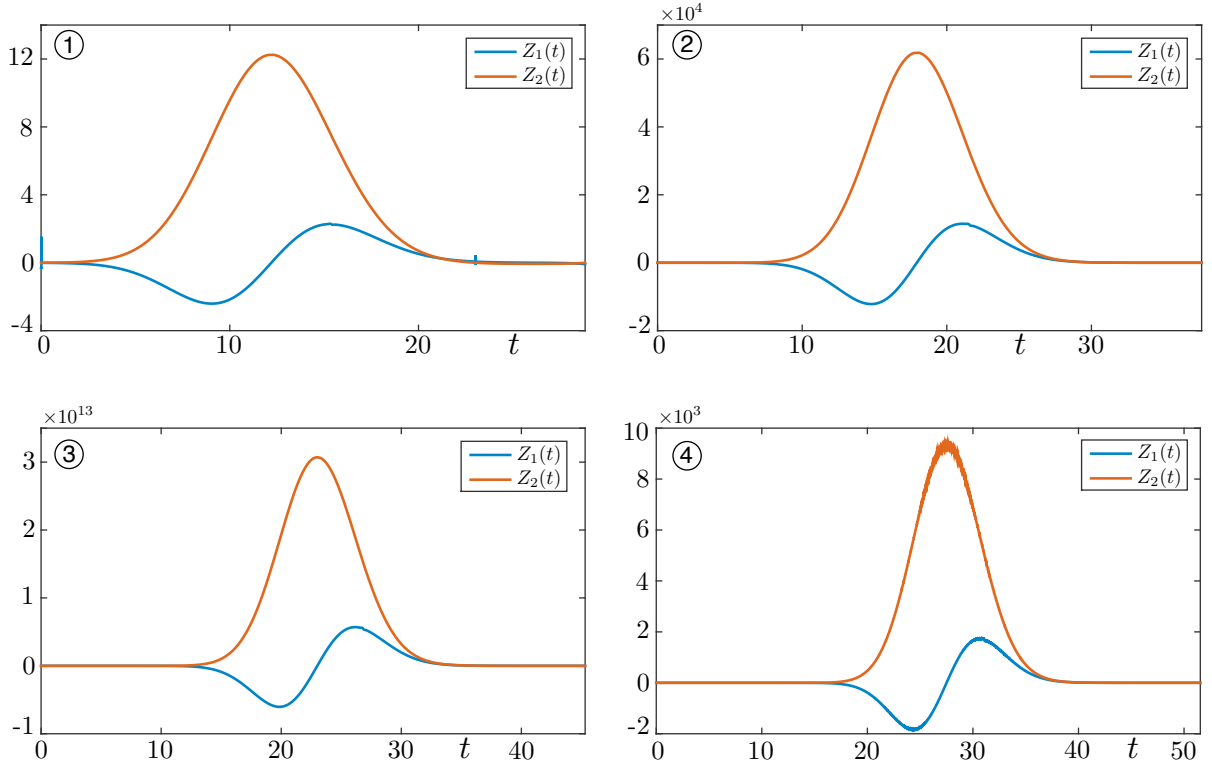

Figure A.15: Adjoint solutions for the headless canard cycles shown in Figure 1 computed analytically using formula A.8. Blue curve: $Z_{1}$. Red curve: $Z_{2}$.

Condition A.11 gives a one-parameter family of suitable linear combinations, one can apply a normalisation to obtain a uniquely defined periodic solution to the adjoint equation.

Simulations of the analytical results from Appendix A. In order to compute the solutions of the adjoint equation given by (A.8) with the two different solutions to the linearized equation $\mathrm{A} .2$, namely $\gamma^{\prime}$ and $(v, w)$, we need to evaluate numerically the function $u$ given by the integral formula A.4, and we also need to evaluate the prefactor

$$
P_{f}(t)=\exp \left(\int_{0}^{t} f^{\prime}\left(\gamma_{1}(s)\right) d s\right) .
$$

To do so, a simple way is to write $u$ as the solution of a second-order differential equation, and $P_{f}$ as the solution of a first-order differential equation, and solve these equations numerically with, e.g., an Euler scheme. More precisely, we 
have

$$
\begin{aligned}
u^{\prime}(t) & =\frac{\exp \left(-\int_{0}^{t} f^{\prime}\left(\gamma_{1}(s)\right) d s\right)}{\gamma_{1}^{\prime}(t)^{2}}:=h(t) \\
h^{\prime}(t) & =-\left(f^{\prime}\left(\gamma_{1}(t)\right)+2 \frac{\gamma_{1}^{\prime \prime}(t)}{\gamma_{1}^{\prime}(t)}\right) h(t) .
\end{aligned}
$$

Similarly, we have

$$
P_{f}^{\prime}(t)=f^{\prime}\left(\gamma_{1}(t)\right) P_{f}(t) .
$$

625 this formula. The reason for this can be understood by looking the expression in A.13 which is singular when $\gamma_{1}^{\prime}(t)=0$, that is, at extrema of $\gamma_{1}$. We can try to integrate these equations by splitting the solution into two branches excluding the extrema. With this strategy, our formula can be used to compute 645 adjoints for all canard cycles and, hence, extend Izhikevich's approach. The second drawback of formula A.8 is that it assumes a Liénard form for the 
system under consideration. Hence, it is not directly applicable to more general planar slow-fast systems, in particular, to biophysical neuron models such as the $2 \mathrm{D}$ reduction of the $\mathrm{HH}$ system that we considered in Section 2.2 .2 .

\section{References}

[1] B. Ermentrout, N. Kopell, Frequency plateaus in a chain of weakly coupled oscillators, SIAM J. Math. Anal. 15 (1984) 215-237.

[2] C. van Vreeswijk, L. Abbot, B. Ermentrout, When inhibition not excitation synchronizes neural firing, J. Comput. Neurosci. 1 (1994) 313-321.

[3] B. Ermentrout, D. Terman, Mathematical Foundations of Neuroscience, Springer, 2010.

[4] F. Hoppenteadt, E. Izhikevich, Weakly Connected Neuroal Networks, Springer, 1997.

[5] N. W. Schulthesis, A. A. Prinz, R. J. Butera (Eds.), Phase Response Curves in Neuroscience: Theory, Experiment, and Analysis, Vol. 6 of Springer series in computational neuroscience, Springer, New York, 2012.

[6] P. Ashwin, S. Coombes, R. Nicks, Mathematical framework for oscillatory network dynamics in neuroscience, J. Nonlinear Sci. 6 (2) (2016) 1-92.

[7] I. Malkin, Methods of Poincare and Liapunov in Theory of Non-Linear Oscillations, Gostexizdat, 1949.

[8] I. Malkin, Some Problems in Nonlinear Oscillation Theory, Gostexizdat, 1956.

[9] A. Buică, J.-P. Françoise, J. Llibre, Periodic solutions of nonlinear periodic differential systems with a small parameter, Commun. Pure Appl. Math. 6 (1) (2007) 103-111.

[10] M. Roseau, Vibrations nonlinéaires et théorie de la stabilité, SpringerVerlag, Berlin, 1966. 
[11] J.-P. Françoise, Oscillations en biologie: Analyse qualitative et modèles, Springer-Verlag, 2005.

675 [12] S. M. Crook, B. Ermentrout, J. M. Bower, Spike frequency affects the synchronization properties of networks of cortical oscillators, Neural Comp. 10 (1998) 1643-1678.

[13] C. C. Chow, N. Kopell, Dynamics of spiking neurons with electrical coupling, Neural Comp. 12 (2000) 1643-1678.

[14] T. Lewis, J. Rinzel, Dynamics of spiking neurons connected by both inhibitory and electrical coupling, J. Comput. Neurosci. 14 (2003) 283-309.

[15] A. Urban, B. Ermentrout, Formation of antiwaves in gap-junction-coupled chains of neurons, Phys. Rev. E 86 (2012) 011907.

[16] M. Feingold, D. L. Gonzalez, O. Piro, H. Vitturo, Phase locking, period doubling, and chaotic phenomena in externally driven excitable systems, Phys. Rev. A 37 (10) (1988) 4060-4063.

[17] D. G. Aronson, B. Ermentrout, N. Kopell, Amplitude response of coupled oscillators, Physica D 41 (1990) 403-449.

[18] B. Ermentrout, N. Kopell, Mechanisms of phase-locking and frequency control in pairs of coupled neural oscillators, in: B. Fiedler (Ed.), Handbook of Dynamical Systems II: Towards Applications, Elsevier, 2002, pp. 5-54.

[19] B. Ermentrout, Type I membranes, phase resetting curves and synchrony, Neural Comp. 8 (1996) 979-1001.

[20] D. Hansel, G. Mato, C. Meunier, Synchrony in excitatory neural networks, Neural Comp. 7 (1995) 307-337.

[21] B. Ermentrout, M. Pascal, B. Gutkin, The effects of spike frequency adaptation and negative feedback on the synchronization of neural oscillators, Neural Comp. 13 (2001) 1285-1310. 
[22] I. Z. Kiss, Y. Zhai, J. L. Hudson, Predicting mutual entrainment of oscillators with experiment-based phase models, Phys. Rev. Lett. 94 (2005) 248301.

[23] M. Wickramasinghe, I. Z. Kiss, Spatially organized dynamical states in chemical oscillator networks: Synchronization, dynamical differentiation, and chimera patterns, PLoS ONE 8 (11) (2013) e80586.

[24] J. Belair, P. Holmes, On linearly coupled relaxation oscillators, Quart. Appl. Math. 42 (1984) 193-219.

[25] T. Bem, J. Rinzel, Short duty cycle destabilizes a half-center oscillator, but gap junctions can restabilize the anti-phase pattern, J. Neurophysiol 91 (2004) 693-703.

[26] T. Chakraboty, R. Rand, The transitions from phase locking to drift in a system of two weakly coupled van der Pol oscillators, Int. J. Nonlinear Mechanics 23 (56) (1988) 369-376.

[27] G. Cymbalyuk, E. Nikolaev, R. Borisyuk, In-phase and antiphase selfoscillations in a model of two electrically coupled pacemakers, Biol. Cybern. 71 (1994) 153-160.

[28] A. Sherman, J. Rinzel, Rhythmogenic effects of weak electrotonic coupling in neuronal models, Proc. Natl. Acad. Sci. USA 89 (1992) 2471-2474.

[29] N. Kopell, D. Somers, Anti-phase solutions in relaxation oscillators coupled through excitatory interactions, J. Math. Biol. 33 (1995) 261-280.

[30] D. Terman, E. Lee, J. Rinzel, T. Bem, Stability of anti-phase and in-phase locking by electrical coupling but not fast inhibition alone, SIAM J. Appl. Dyn. Syst. 10 (3) (2011) 1127-1153.

[31] B. Pfeuty, G. Mato, D. Golomb, D. Hansel, The combined effects of inhibitory and electrical synapses in synchrony, Neural Comp. 17 (2005) 633670. 
[32] J. Mancilla, T. Lewis, D. Pinto, J. Rubin, C. B.W., Synchronization of electrically coupled pairs of inhibitory interneurons in neocortex, J. Neurosci. 27 (8) (2007) 2058-2073.

[33] S. Coombes, Phase-locking in networks of pulse-coupled McKean relaxation oscillators, Phys. D 160 (2001) 173-188.

[34] S. Coombes, Neuronal networks with gap junctions: A study of piecewise linear planar neuron models, SIAM J. Appl. Dyn. Syst. 7 (3) (2008) 11011129.

[35] E. Benoît, J.-L. Callot, F. Diener, M. Diener, Chasse au canard, Collect. Math. 32 (1-2) (1981) 37-119.

[36] M. Krupa, P. Szmolyan, Relaxation oscillation and canard explosion, J. Differential Equations 174 (2) (2001) 312-368.

[37] M. Brøns, Bifurcations and instabilities in the Greitzer model for compressor system surge, Math. Eng. Ind. 2 (1988) 51-63.

[38] M. Krupa, M. Wechselberger, Local analysis near a folded saddle-node singularity, J. Differ. Equ. 248 (2010) 2841-2888.

[39] J. Guckenheimer, K. Hoffman, W. Weckesser, Numerical computation of canards, Int. J. Bifur. and Chaos 10 (12) (2000) 2669-2687.

[40] M. Desroches, J. Guckenheimer, B. Krauskopf, C. Kuehn, H. M. Osinga, M. Wechselberger, Mixed-mode oscillations in a multiple time scale, SIAM Rev. 54 (2012) 211-288.

[41] M. Desroches, T. J. Kaper, M. Krupa, Mixed-mode bursting oscillations: Dynamics created by a slow passage through spike-adding canard explosion in a square-wave burster, Chaos 23 (2013) 046106.

[42] T. Vo, R. Bertram, M. Wechselberger, Multiple geometric viewpoints of mixed mode dynamics associated with pseudo-plateau bursting, SIAM J. Appl. Dyn. Syst. 12 (2) (2013) 789-830. 
[43] M. Krupa, N. Popovic, N. Kopell, H. G. Rotstein, Mixed-mode oscillations in a three time-scale model for the dopaminergic neuron, Chaos 18 (49) (2008) 015106.

[44] H. G. Rotstein, T. Oppermann, J. A. White, N. Kopell, The dynamic structure underlying subthreshold oscillatory activity and the onset of spikes in a model of medial entorhinal cortex stellate cells, J. Comput. Neurosci. 21 (2006) 271-292.

[45] J. Rubin, M. Wechselberger, Giant squid-hidden canard: the 3D geometry of the Hodgkin-Huxley model, Biol. Cybern. 97 (2007) 5-32.

[46] J. Drover, J. Rubin, J. Su, B. Ermentrout, Analysis of a canard mechanism by which excitatory synaptic coupling can synchronize neurons at low firing frequencies, SIAM J. Appl. Dyn. Syst. 65 (1) (2004) 69-92.

[47] B. Ermentrout, M. Wechselberger, Canards, clusters and synchronization in a weakly coupled interneuron model, SIAM J. Appl. Dyn. Syst. 8 (2009) $253-278$.

[48] K.-L. Roberts, J. Rubin, M. Welchselberger, Averaging, folded singularities, and torus canards: Explaining transitions between bursting and spiking in a coupled neuron model, SIAM J. Appl. Dyn. Syst. 14 (4) (2015) $1808-1844$.

[49] H. G. Rotstein, N. Kopell, A. M. Zhabotinsky, I. R. Epstein, A canard mechanism for localization in systems of globally coupled oscillators, SIAM J. Appl. Math. 63 (6) (2003) 1998-2019.

[50] H. G. Rotstein, R. Kuske, Localized and asynchronous patterns via canards in coupled calcium oscillators, Phys. D 215 (2006) 46-61.

[51] E. Köksal Ersöz, M. Desroches, M. Krupa, F. Clément, Canard-mediated (de)synchronization in coupled phantom bursters, SIAM J. Appl. Dyn. Syst. 15 (1) (2016) 580-608. 
[52] E. Brown, J. Moehlis, P. Holmes, On the phase reduction and response dynamics of neural oscillator populations, Neural Comp. 16 (2003) 673715.

[53] E. M. Izhikevich, Phase equations for relaxation oscillators, SIAM J. Appl. Math. 60 (5) (2000) 1789-1805.

[54] R. Curtu, A. Shpiro, N. Rubin, J. Rinzel, Mechanisms for frequency control in neural competition models, SIAM J. Appl. Dyn. Syst. 7 (2) (2008) 609649.

[55] C. Zhang, T. Lewis, Phase response properties of half-center oscillators, J. Comput. Neurosci. 35 (2013) 55-74.

[56] T. Netoff, C. Acker, J. Bettencourt, J. A. White, Beyond two-cell networks: experimental measurement of neuronal responses to multiple synaptic inputs, J. Comput. Neurosci. 18 (2005) 287-295.

[57] Y. Kuramoto, Chemical oscillators, waves, and turbulence, Springer-Verlag, 1984.

[58] B. Ermentrout, Simulating, analyzing, and animating dynamical systems: a guide to XPPAUT for researchers and students, SIAM, 2002.

[59] E. M. Izhikevich, F. C. Hoppensteadt, Slowly coupled oscillators: phase dynamics and synchronization, SIAM J. Appl. Math. 63 (6) (2003) 19351953.

[60] W. Govaerts, B. Sautois, Computation of the phase response curve: A direct numerical approach, Neural Comp. 18 (4) (2006) 817-847.

[61] E. J. Doedel, A. R. Champneys, F. Dercole, T. Fairgrieve, Y. Kuznetsov, B. E. Oldeman, R. Paffenroth, B. Sandstede, X. J. Wang, Auto-07P : Continuation and bifurcation software for ordinary differential equations, available at http://cmvl.cs.concordia.ca/auto (2007). 
[62] B. Ermentrout, B. Beverlin II, T. Netoff, Phase response curves to measure ion channel effects on neurons, in: N. W. Schultheiss, A. A. Prinz, R. J. Butera (Eds.), Phase response curves in neuroscience, Springer, 2012, pp. 207-236.

[63] J. Moehlis, Canards for a reduction of Hodgkin-Huxley equations, J. Math. Biol. 52 (2006) 141-153.

[64] P. De Maesschalck, M. Desroches, Numerical continuation techniques for planar slow-fast systems, SIAM J. Appl. Dyn. Syst. 12 (3) (2013) 11591180.

[65] P. Ashwin, J. Swift, The dynamics of $n$ weakly coupled identical oscillators, J. Nonlinear Sci. 2 (1992) 69-108.

[66] D. Somers, N. Kopell, Rapid transition though fast threshold modulation, Biol. Cybern. 68 (1993) 393-407.

[67] E. Lee, D. Terman, Stable antiphase oscillations in a network of electrically coupled neurons, SIAM J. Appl. Dyn. Syst. 12 (1) (2013) 1-27.

[68] M. Oh, V. Matveev, Loss of phase-locking in non-weakly coupled inhibitory networks of type-I model neurons, J. Comput. Neurosci. 26 (2009) 303-320.

[69] S. Maran, C. Canavier, Using phase resetting to predict 1:1 and 2:2 locking in two neuron networks in which firing order is not always preserved, J. Comput. Neurosci. 24 (2008) 37-55.

[70] A. T. Winfree, The geometry of biological time, Vol. 12 of Interdisciplinary Applied Mathematics, Springer, 1980.

[71] P. Langfield, B. Krauskopf, H. M. Osinga, Solving Winfree's puzzle: The isochrons in the FitzHugh-Nagumo model, Chaos 24 (2014) 013131.

${ }_{830}$ [72] P. Langfield, B. Krauskopf, H. M. Osinga, Forward-time and backwardtime isochrons and their interactions, SIAM J. Appl. Dyn. Syst. 14 (3) (2015) 1418-1453. 
[73] G. Ermentrout, Period doublings and possible chaos in neural models, SIAM J. Appl. Math. 44 (1) (1984) 80-95.

835

[80] E. A. Coddington, N. Levinson, Theory of ordinary differential equations, McGraw-Hill, 1987.

[81] S. Schecter, Adjoint equation and Melnikov function, unpublished note available at http://www4.ncsu.edu/ schecter/ma_732_sp13/melnikov.pdf. 